\title{
Review Article \\ Stochastic Comparisons of Order Statistics and Spacings: A Review
}

\author{
Subhash Kochar \\ Fariborz Maseeh Department of Mathematics and Statistics, Portland State University, Portland, \\ OR 97201, USA \\ Correspondence should be addressed to Subhash Kochar, kochar@pdx.edu \\ Received 13 September 2012; Accepted 3 October 2012 \\ Academic Editors: C. Fox, J. López-Fidalgo, O. Pons, and C. Proppe \\ Copyright (C) 2012 Subhash Kochar. This is an open access article distributed under the Creative \\ Commons Attribution License, which permits unrestricted use, distribution, and reproduction in \\ any medium, provided the original work is properly cited. \\ We review some of the recent developments in the area of stochastic comparisons of order statistics \\ and sample spacings. We consider the cases when the parent observations are identically as well \\ as nonidentically distributed. But most of the time we will be assuming that the observations are \\ independent. The case of independent exponentials with unequal scale parameters as well as the \\ proportional hazard rate model is discussed in detail.
}

\section{Introduction}

The simplest and the most popular method of comparing the magnitudes of two random variables is through their means and medians. It may happen that in some cases the median of $X$ is larger than that of $Y$, while the mean of $X$ is smaller than the mean of $Y$. However, this confusion will not arise if the random variables are stochastically ordered. Similarly, the same may happen if one would like to compare the variability of $X$ with that of $Y$ based only on numerical measures like standard deviation, and so forth. Besides, these characteristics of distributions might not exist in some cases. In most cases one can express various forms of knowledge about the underlying distributions in terms of their survival functions, quantile functions, hazard rate functions, mean residual functions, and other suitable functions of probability distributions. These methods are much more informative than those based only on few numerical characteristics of distributions. Comparisons of random variables based on such functions usually establish partial orders among them. We call them as stochastic orders.

Stochastic models are usually sufficiently complex in various fields of statistics. Obtaining bounds and approximations for their characteristics is of practical importance. That is, the approximation of a stochastic model either by a simpler model or by a model 
with simple constituent components might lead to convenient bounds and approximations for some particular and desired characteristics of the model. The study of changes in the properties of a model, as the constituent components vary, is also of great interest. Accordingly, since the stochastic components of models involve random variables, the topic of stochastic orders among random variables plays an important role in these areas. Books by Muller and Stoyan [1] and Shaked and Shanthikumar [2] give excellent treatment of this topic.

Order statistics and spacings are of great interest in many areas of statistics and they have received a lot of attention from many researchers. Let $X_{1}, \ldots, X_{n}$ be $n$ random variables. The $i$ th order statistic, the $i$ th smallest of $X_{i}{ }^{\prime} s$, is denoted by $X_{i: n}$. In reliability engineering, an $n$ component system that works if and only if at least $k$ of the $n$ components work is called a $k$-out-of- $n$ system. The lifetime of a $k$-out-of- $n$ system can be represented as $X_{n-k+1: n}$. A parallel system is a 1-out-of- $n$ system while a series system is an $n$-out-of- $n$ system. Thus, the study of lifetimes of $k$-out-of- $n$ systems is equivalent to the study of the stochastic properties of order statistics. Spacings, the differences between successive order statistics, and their functions are also important in statistics, in general, and in particular in the context of life testing and reliability models. The books by David and Nagaraja [3], and Arnold et al. [4]; and two volumes of papers on this topic by Balakrishnan and Rao $[5,6]$ are excellent sources of information on this topic. But most of this work has been confined to the case when the observations are independent and identically distributed (i.i.d.). In many practical situations, like in reliability theory, the observations are not necessarily i.i.d. Only during the last two decades or so this topic has got the attention of researchers. Some important early references for this case are Sen [7], David and Nagaraja [3], Shaked and Tong [8], Bapat and Beg [9], Boland et al. [10], Kochar [11], and Nappo and Spizzichino [12], Boland et al. [13] and Balakrishnan [14], among others.

Some interesting partial ordering results on order statistics and spacings from independent but nonidentically random variables have been obtained by Pledger and Proschan [15], Proschan and Sethuraman [16], Bapat and Kochar [17], Boland et al. [18], Kochar and Kirmani [19], Kochar and Korwar [20], Kochar and Rojo [21], Dykstra et al. [22], Kochar and Ma [23], Bon and Păltănea [24], Kochar [25], Khaledi and Kochar [26-30]. The book by Shaked and Shanthikumar [2] gives an excellent description of the various results on this topic till 2007.

In this review paper, we will discuss some latest developments on the topic of stochastic comparisons of order statistics and spacings. In Section 2, we introduce the required notation and definitions. Sections 3 and 4 are devoted to stochastic comparisons of order statistics in one-sample and two-sample problems, respectively. In Section 5, we discuss the topic of stochastic orderings among spacings in one-sample problem and two sample problems. Section 6 is devoted to some applications of these results. Throughout this chapter increasing means nondecreasing and decreasing means nonincreasing; and we will be assuming that all distributions under study are absolutely continuous.

\section{Definitions and Some Preliminaries}

In this section, we recall some basic definitions of stochastic orders and their properties. Let $X$ and $Y$ be univariate random variables with distribution functions $F$ and $G$, survival functions $\bar{F}$ and $\bar{G}$, density functions $f$ and $g$; and hazard rates $r_{F}(=f / \bar{F})$ and $r_{G}(=g / \bar{G})$, respectively. Let $l_{X}\left(l_{Y}\right)$ and $u_{X}\left(u_{Y}\right)$ be the left and the right endpoints of the support of $X(Y)$. 


\subsection{Magnitude Orders}

First we give some definitions of stochastic orders to compare the magnitudes of two random variables.

Definition 2.1. $X$ is said to be stochastically smaller than $Y$ (denoted by $X \leq_{\text {st }} Y$ ) if

$$
\bar{F}(x) \leq \bar{G}(x) \quad \forall x .
$$

It is easy to see that $X \leq_{\text {st }} Y$ if and only if

$$
F^{-1}(u) \leq G^{-1}(u) \quad \forall u \in(0,1)
$$

It can be shown that this is also equivalent to $E[g(X)] \leq E[g(Y)]$ for all increasing function $g$ for which expectations exist.

Definition 2.2. $X$ is said to be smaller than $Y$ in hazard rate ordering (denoted by $X \leq{ }_{h r} Y$ ) if $\bar{G}(x) / \bar{F}(x)$ is increasing in $x \in\left(-\infty, \max \left(u_{X}, u_{Y}\right)\right)$.

It can be shown that $X \leq_{h r} Y$ is equivalent to the inequalities

$$
P[X-t>x \mid X>t] \leq P[Y-t>x \mid Y>t], \quad \forall x \geq 0, t .
$$

In other words, the conditional distributions, given that the random variables are at least of a certain size, are all stochastically ordered (in the standard sense) in the same direction. Thus, if $X$ and $Y$ represent the survival times of different models of an appliance that satisfy this ordering, one model is better (in the sense of stochastic ordering) when the appliances are new, the same appliance is better when both are one month old, and in fact is better no matter how much time has elapsed. It is clearly useful to know when this strong type of stochastic ordering holds since qualitative judgements are then easy to make. In case the hazard rates exist, it is easy to see that $X \leq_{h r} Y$, if and only if, $r_{G}(x) \leq r_{F}(x)$ for every $x$. The hazard rate ordering is also known as uniform stochastic ordering in the literature.

Definition 2.3. $X$ is said to be smaller than $Y$ in the reverse hazard rate order, denoted by $X \leq_{\mathrm{rh}} Y$, if

$$
P(t-X>x \mid X \leq t) \geq P(t-Y>x \mid Y \leq t), \quad \forall x \geq 0 \text { and all } t .
$$

It is shown in Ross [31] that if $X$ and $Y$ are two independent random variables, then $X \leq_{\text {st }} Y$ may not imply

$$
X+(X+Y) \leq_{s t} Y+(X+Y)
$$

However (2.5) will hold if $X$ and $Y$ satisfy a stronger ordering called likelihood ratio ordering as defined below. 
Definition 2.4. $X$ is said to be smaller than $Y$ in likelihood ratio ordering (denoted by $X \leq \operatorname{lr} Y$ ) if $g(x) / f(x)$ is increasing in $x \in\left(l_{X}, u_{X}\right) \cup\left(l_{Y}, u_{Y}\right)$.

The mean residual life function of a random variable $X$ is defined as $\mu_{X}(t)=\int_{x}^{\infty} \bar{F}(t) d t$. An interesting order based on the mean residual life is defined as follows.

Definition 2.5. $X$ is said to be smaller than $Y$ in the mean residual life order, denoted by $X \leq_{\mathrm{mrl}} Y$, if

$$
\mu_{X}(t) \leq \mu_{Y}(t) \quad \forall t
$$

When the supports of $X$ and $Y$ have a common left end-point, we have the following chain of implications among the above stochastic orders:

$$
\begin{aligned}
& X \leq_{\mathrm{lr}} Y \Longrightarrow X \leq_{\mathrm{hr}} Y \Longrightarrow X \leq_{\mathrm{mrl}} Y \\
& \Downarrow \quad \Downarrow \quad \Downarrow \\
& X \leq_{\mathrm{rh}} Y \Longrightarrow X \leq_{\mathrm{st}} Y \Longrightarrow E[X] \leq E[Y] \text {. }
\end{aligned}
$$

Definition 2.6. $X$ is said to be smaller than $Y$ in the increasing convex order (denoted by $X \leq_{\mathrm{icx}} Y$ ) if

$$
\int_{t}^{+\infty} \bar{F}(x) d x \leq \int_{t}^{+\infty} \bar{G}(x) d x, \quad \forall t
$$

Note that (2.8) holds if and only if

$$
E[\phi(X)] \leq E[\phi(Y)]
$$

for every convex function $\phi$ for which the above expectations exist. Also note that $X \leq_{\mathrm{st}} Y \Rightarrow$ $X \leq_{\text {icx }} Y$.

Another order closely related to the likelihood ratio order is the joint likelihood ordering as introduced by Shanthikumar and Yao [32].

Definition 2.7. For a bivariate random variable $(X, Y), X$ is said to be smaller than $Y$ according to joint likelihood ordering, denoted by $X \leq_{\ell r: j}$, if and only if

$$
E[\phi(X, Y)] \geq E[\phi(Y, X)], \quad \phi \in \mathcal{G}_{\ell r},
$$

where

$$
\mathcal{G}_{\ell r}:\{\phi: \phi(y, x) \leq \phi(x, y), x \leq y\} .
$$

It can be seen that

$$
X_{1} \leq_{\ell: j} X_{2} \Longleftrightarrow f \in \mathcal{G}_{\ell r},
$$

where $f(\cdot, \cdot)$ denotes the joint density of $\left(X_{1}, X_{2}\right)$. 
As pointed out by Shanthikumar and Yao [32], joint likelihood ratio ordering between the components of a bivariate random vector may not imply likelihood ratio ordering between their marginal distributions unless the random variables are independent, but it does imply stochastic ordering between them, that is,

$$
X \stackrel{e r: j}{\leq} Y \Longrightarrow X \stackrel{\text { st }}{\leq} Y
$$

A bivariate function $\phi \in \mathcal{G}_{\ell r}$ is called arrangement increasing (AI). Hollander et al. [33] have studied many interesting properties of such functions, though, apparently, they did not relate it to the notion of likelihood ratio ordering.

The above idea can be extended to compare the components of an $n$-dimensional vector $\mathbf{X}=\left(X_{1}, \ldots, X_{n}\right)$. We define $X_{1} \leq_{\ell r: j} \cdots \leq_{\ell r: j} X_{n}$ if the joint density $f\left(x_{1}, \ldots, x_{n}\right)$ of $\mathbf{X}$ is an arrangement increasing function. (See, Marshall et al. [34] for the definition of an arrangement increasing function on $\mathcal{R}^{n}$.)

In a different context, Robertson and Wright [35] studied a subclass of arrangement increasing functions on $\mathcal{R}^{n}$, which they call as ISO* functions, as described below. Let $\mathbf{x}$ and $\mathbf{y}$ be two vectors on $\mathcal{R}^{n}$ such that $\sum_{i=1}^{j} y_{i} \leq \sum_{i=1}^{j} x_{i}, j=1, \ldots, n-1$ and $\sum_{i=1}^{n} y_{i}=\sum_{i=1}^{n} x_{i}$. We will denote this partial ordering between the vectors by $\mathbf{x} \stackrel{\star}{\ll} \mathbf{y}$.

Definition 2.8. A real-valued function $\phi$ defined on a set $\mathcal{A} \subset \mathbb{R}^{n}$ is said to be ISO* on $\mathcal{A}$ if $\phi(\mathbf{x}) \leq \phi(\mathbf{y})$, for all $\mathbf{x} \stackrel{\star}{\ll} \mathbf{y}$.

As mentioned earlier, an $\mathrm{ISO}^{*}$ function is arrangement increasing but the converse is not true. It is easy to see that the joint density $f\left(x_{1}, x_{2}\right)$ of a bivariate random vector $\left(X_{1}, X_{2}\right)$ is ISO $^{*}$ if and only if the conditional density of $X_{2}$ given $X_{1}+X_{2}=t$ is monotonically increasing for each fixed $t$.

The usual likelihood ratio order has the following multivariate version.

Definition 2.9. Let $\mathbf{X}=\left(X_{1}, X_{2}, \ldots, X_{n}\right)$ and $\mathbf{Y}=\left(Y_{1}, Y_{2}, \ldots, Y_{n}\right)$ be two $n$-dimensional random vectors with absolutely continuous (or discrete) distribution functions and let $f$ and $g$ denote their continuous (or discrete) density functions, respectively. Suppose that

$$
f(\mathbf{x}) g(\mathbf{y}) \leq f(\mathbf{x} \wedge \mathbf{y}) g(\mathbf{x} \vee \mathbf{y})
$$

for every $\mathbf{x}$ and $\mathbf{y}$. Then $X$ is said to be smaller than $Y$ in the multivariate likelihood ratio order (denoted as $\mathbf{X} \leq \operatorname{lr} \mathbf{Y}$ ).

Definition 2.10. The random vector $\mathbf{X}=\left(X_{1}, \ldots, X_{n}\right)$ is smaller than the random vector $\mathbf{Y}=$ $\left(Y_{1}, \ldots, Y_{n}\right)$ in the multivariate stochastic order (denoted by $\left.\mathbf{X} \stackrel{\text { st }}{\leq} \mathbf{Y}\right)$ if $h(\mathbf{X}) \leq_{\text {st }} h(\mathbf{Y})$ for all increasing functions $h$.

It is known that multivariate likelihood ratio ordering implies multivariate stochastic ordering, but the converse is not true. Also if two random vectors are ordered according to multivariate likelihood ratio (stochastic) ordering, then their corresponding subsets of components are also ordered accordingly. For more details on stochastic orderings, see Chapters 1 and 4 of Shaked and Shanthikumar [2]. 


\subsection{Variability Orders}

One of the basic criteria for comparing variability in probability distributions is that of dispersive ordering. Let $F^{-1}$ and $G^{-1}$ be the right continuous inverses (quantile functions) of $F$ and $G$, respectively.

Definition 2.11. $X$ is less dispersed than $Y$ (denoted by $X \leq_{\operatorname{disp}} Y$ ) if

$$
F^{-1}(\beta)-F^{-1}(\alpha) \leq G^{-1}(\beta)-G^{-1}(\alpha), \quad \forall 0 \leq \alpha \leq \beta \leq 1 .
$$

Note that (2.15) is equivalent to

$$
G^{-1} F(x)-x \text { increasing in } x \text {. }
$$

When (2.16) holds, Doksum [36] called this ordering as tail-ordering and used it to find bounds on powers and efficiencies of nonparametric tests. Deshpande and Kochar [37] pointed out that tail ordering is same as dispersive ordering and obtained some new results for this partial order.

A consequence of $X \leq_{\text {disp }} Y$ is that $\left|X_{1}-X_{2}\right| \leq_{s t}\left|Y_{1}-Y_{2}\right|$ and which in turn implies $\operatorname{var}(X) \leq \operatorname{var}(Y)$ as well as $E\left[\left|X_{1}-X_{2}\right|\right] \leq E\left[\left|Y_{1}-Y_{2}\right|\right]$, where $X_{1}, X_{2}\left(Y_{1}, Y_{2}\right)$ are two independent copies of $X(Y)$. For details, see Saunders [38], Saunders and Moran [39], Lewis and Thompson [40], Deshpande and Kochar [37], Bagai and Kochar [41], Bartoszewicz $[42,43]$, and Section 2.B of Shaked and Shanthikumar [2].

\section{Connection between Hazard Rate Order and Dispersive Order}

Kochar [44] observed that

$$
\mathrm{X} \leq_{\mathrm{hr}} Y \Longrightarrow P[X X Y Y]+P[Y X X Y] \leq P[Y Y X X]+P[X Y Y X]
$$

where, for example, $P[X X Y Y]$ means $P\left[X_{1: 2} \leq X_{2: 2} \leq Y_{1: 2} \leq Y_{2: 2}\right]$ with the notation that $Z_{i: n}$ denotes the $i$ th order statistic of a random sample $Z_{1}, \ldots, Z_{n}$ on $Z$.

Equation (2.17) indicates that hazard rate ordering not only compares the magnitudes of two random variables, but it also has perhaps some connection with the variability between the random variables. On differentiating (2.16), one can easily see that

$$
X \leq_{\text {disp }} Y \Longleftrightarrow r_{G}\left(G^{-1}(u)\right) \leq r_{F}\left(F^{-1}(u)\right) \quad \forall u \in(0,1),
$$

when the random variables $X$ and $Y$ admit densities. This lead Bagai and Kochar [41] to prove the following connection between hazard rate ordering and dispersive ordering. 
Theorem 2.12. (a) If $X$ or $Y$ have increasing failure rate (IFR), then $X \leq_{\text {disp }} \Rightarrow X \leq_{h r} Y$.

(b) If $X$ or $Y$ have decreasing failure rate (DFR), then $X \leq_{h r} \Rightarrow X \leq_{\text {disp }} Y$.

For a review on applications of dispersive ordering, see Joen et al. [45].

A weaker variability ordering is right spread order as introduced in Fernandez-Ponce et al. [46]. It was also independently studied by Shaked and Shanthikumar[47] who call it as excess wealth order.

Definition 2.13. $X$ is said to be smaller than $Y$ in the excess wealth order (denoted by $X \leq_{\mathrm{ew}} Y$ ) if

$$
\int_{F^{-1}(p)}^{\infty} \bar{F}(x) d x \leq \int_{G^{-1}(p)}^{\infty} \bar{G}(x) d x, \quad 0 \leq p \leq 1 .
$$

Dispersive ordering implies excess wealth ordering which in turn implies that the commonly used measures of variability like variances are ordered. One may refer to Shaked and Shanthikumar [2] for a comprehensive discussion of this order.

\subsection{Skewness Orderings}

Skewness describes the departure of a distribution from symmetry, where one tail of the density is more "stretched out" than the other. Several partial orders have been introduced in the literature to compare the relative skewness of probability distributions. van Zwet [48] introduced the concept of convex transform order to compare two distributions according to skewness as defined below.

Definition 2.14. $X$ is said to be smaller than $Y$ in the convex transform order, denoted by $X \leq_{c} Y$ if and only if, $G^{-1} F(x)$ is convex in $x$ on the support of $X$.

If $X \leq_{c} Y$, then $Y$ is more skewed than $X$ as explained in van Zwet [48] and Marshall et al. [34]. The convex transform order is also called more IFR (increasing failure rate) order in reliability theory, since when $f$ and $g$ exist, the convexity of $G^{-1} F(x)$ means that

$$
\frac{f\left(F^{-1}(u)\right)}{g\left(G^{-1}(u)\right)}=\frac{r_{X}\left(F^{-1}(u)\right)}{r_{Y}\left(G^{-1}(u)\right)}
$$

is increasing in $u \in[0,1]$. Thus $X \leq_{c} Y$ can be interpreted to mean that $X$ ages faster than $Y$ in some sense. Gamma distributions are ordered according to the convex transform order in terms of their shape parameters.

Another well-known partial order to compare the skewness of two probability distributions is star order.

Definition 2.15. $X$ is said to be smaller than $Y$ in the star order, denoted by $X \leq_{\star} Y$ (or $F \leq_{\star} G$ ) if the function $G^{-1} F(x)$ is star shaped in the sense that $G^{-1} F(x) / x$ is increasing in $x$ on the support of $X$. 
The star order is also called more IFRA (increasing failure rate in average) order in reliability theory, since the average failure of $F$ at $x$ is

$$
\bar{r}_{X}(x)=\frac{1}{x} \int_{0}^{x} r_{X}(u) d u=\frac{-\ln \bar{F}(x)}{x} .
$$

Thus $F \leq_{\star} G$ can be interpreted in terms of average failure rates as

$$
\frac{\bar{r}_{X}\left(F^{-1}(u)\right)}{\bar{r}_{Y}\left(G^{-1}(u)\right)}
$$

is increasing in $u \in(0,1]$. Note that $X$ has an increasing failure rate if and only if $F$ is starordered with respect to exponential distribution.

The function

$$
L_{X}(u)=\frac{1}{E(X)} \int_{0}^{F^{-1}(u)} x d F(x)
$$

is known as Lorenz curve in the economics literature. It is often used to express inequality in incomes. Based on Lorenz curve, the Lorenz order has been proposed in Economics to compare income inequalities.

Definition 2.16. $X$ is said to be smaller than $Y$ in the Lorenz order, denoted by $X \leq_{\text {Lorenz }} Y$, if

$$
\frac{1}{E(X)} \int_{0}^{F^{-1}(u)} x d F(x) \geq \frac{1}{E(Y)} \int_{0}^{G^{-1}(u)} x d G(x), \quad \forall u \in(0,1]
$$

It is known in the literature (Marshall et al. [34] ) that,

$$
X \leq_{c} Y \Longrightarrow X \leq_{\star} Y \Longrightarrow X \leq_{\text {Lorenz }} Y \Longrightarrow \mathrm{cv}(X) \leq \mathrm{cv}(Y),
$$

where $\mathrm{cv}(X)=\sqrt{\operatorname{Var}(x) / E(X)}$ denote the coefficient of variation of $X$.

All the above partial orders are scale invariant. A good discussion of the star order and Lorenz order can be found in Barlow and Proschan [49], Marshall et al. [34], and Kochar [50].

\subsection{Dependence Concepts and Orderings}

Let $(X, Y)$ be a bivariate ramdom vector with joint distribution $F(x, y)$ and with density function $f(x, y)$. Lehmann [51] introduced many partial orderings for dependence.

Definition 2.17. (a) $X$ and $Y$ are $\mathrm{TP}_{2}$ dependent or likelihood dependent if their joint density $f(x, y)$ is totally positive of order 2 in $x$ and $y$, or more precisely if

$$
\left|\begin{array}{ll}
f\left(x_{1}, y_{1}\right) & f\left(x_{1}, y_{2}\right) \\
f\left(x_{2}, y_{1}\right) & f\left(x_{2}, y_{2}\right)
\end{array}\right| \geq 0
$$

whenever $x_{1}<x_{2}, y_{1}<y_{2}$. 
(b) $Y$ is stochastically increasing in $X$, denoted by $\operatorname{SI}(Y \mid X)$, if $P(Y>y \mid X=x)$ is increasing in $x$ for all $y$, or equivalently,

$$
P(Y \leq y \mid X=x) \geq P\left(Y \leq y \mid X=x^{*}\right), \quad x \leq x^{*}
$$

(c) $Y$ is right tail increasing in $X$, denoted by $\operatorname{RTI}(Y \mid X)$, if $P(Y>y \mid X>x)$ is increasing in $x$ for all $y$.

(d) $Y$ is left tail decreasing in $X$, denoted by $\operatorname{LTD}(Y \mid X)$, if $P(Y \leq y \mid X \leq x)$ is decreasing in $x$ for all $y$.

Finally, random variables $X$ and $Y$ are associated (written $A(X, Y)$ ) if $\operatorname{cov}[\Gamma(X, Y)$, $\triangle(X, Y)] \geq 0$ for all pairs of increasing binary functions $\Gamma$ and $\triangle$. As shown in Barlow and Proschan [49, page 143] the following chain of implications holds among the above notions of positive dependence:

$$
\mathrm{TP}_{2}(X, Y) \Longrightarrow \mathrm{SI}(X \mid Y) \Longrightarrow \operatorname{RTI}(X \mid Y) \Longrightarrow A(S, T) \Longrightarrow \operatorname{Cov}(X, Y) \geq 0
$$

These concepts of bivariate dependence can be easily extended to the multivariate case. A function $\psi: R^{n} \rightarrow[0, \infty)$ is said to be multivariate total positivity of order 2 (denoted by $\left.\mathrm{MTP}_{2}\right)$ if

$$
\psi(\mathbf{x}) \psi(\mathbf{y}) \leq \psi(\mathbf{x} \wedge \mathbf{y}) \psi(\mathbf{x} \vee \mathbf{y}) \quad \text { for every } \mathbf{x} \text { and } \mathbf{y} \text { in } R^{n},
$$

where $\mathbf{x} \wedge \mathbf{y}=\left(\min \left(x_{1}, y_{1}\right), \ldots, \min \left(x_{n}, y_{n}\right)\right)$ and $\mathbf{x} \vee \mathbf{y}=\left(\max \left(x_{1}, y_{1}\right), \ldots, \max \left(x_{n}, y_{n}\right)\right)$. Random variables $X_{1}, \ldots, X_{n}$ are said to be $\mathrm{MTP}_{2}$ dependent if their joint density function is $\mathrm{MTP}_{2}$. It is shown in Kemperman [52] (see also Block and Ting, [53]) that if the support of a random vector $\mathbf{X}=\left(X_{1}, \ldots, X_{n}\right)$ is a lattice (i.e., if $\mathbf{x}$ and $\mathbf{y}$ are in the support of $\mathbf{X}$ then so are $\mathbf{x} \wedge \mathbf{y}$ and $\mathbf{x} \vee \mathbf{y}$ ) then $X$ is $\mathrm{MTP}_{2}$ if and only if, its density function $f$ is $\mathrm{TP}_{2}$ in each pair of its variables when the other $(n-2)$ variables are held fixed. See Karlin and Rinott [54] for more details on properties of $\mathrm{MTP}_{2}$ functions. Also refer to Joe [55] and Nelsen [56] for a comprehensive discussion.

Observing that when $X$ and $Y$ are continuous, inequality (2.27) can be written as

$$
H_{\left[\xi_{q}\right]} \circ H_{\left[\xi_{q}\right]}^{-1}(u) \leq u,
$$

where $\xi_{p}=F^{-1}(p)$ stands for the $p$ th quantile of the marginal distribution of $X$, and $H_{[s]}$ denotes the conditional distribution of $Y$ given $X=s$. Averous et al. [57] proposed the following definition to measure the relative degree of monotone dependence between two pairs of bivariate random variables $\left(X_{1}, Y_{1}\right)$ and $\left(X_{2}, Y_{2}\right)$. 
Definition 2.18. $Y_{1}$ is said to be less stochastic increasing in $X_{1}$ than $Y_{2}$ is in $X_{2}$, denoted by $\left(Y_{1} \mid X_{1}\right) \preceq_{\text {SI }}\left(Y_{2} \mid X_{2}\right)$, if and only if, for $0 \leq u \leq 1$, and $0<p \leq q<1$,

$$
H_{2\left[\tilde{\xi}_{2 q}\right]} \circ \mathrm{H}_{2\left[\xi_{2} p\right]}^{-1}(u) \leq H_{1\left[\xi_{1 q}\right]} \circ H_{1\left[\xi_{1} p\right]}^{-1}(u),
$$

where $\xi_{i p}=F_{i}^{-1}(p)$ stands for the $p$ th quantile of the marginal distribution of $X_{i}$, and $H_{i[s]}$ denotes the conditional distribution of $Y_{i}$ given $X_{i}=s$, for $i=1,2$.

Dolati et al. [58] proposed the following weaker dependence order based on RTI criteria, called more RTI order.

Definition 2.19. $Y_{1}$ is said to be less right-tail increasing (RTI) in $X_{1}$ than $Y_{2}$ is in $X_{2}$, denoted by $\left(Y_{1} \mid X_{1}\right) \leq$ RTI $\left(Y_{2} \mid X_{2}\right)$, if and only if, for $0 \leq u \leq 1$, and $0<p \leq q<1$,

$$
H_{2\left[\xi_{2 q}\right]}^{*} \circ H_{2\left[\xi_{2 p}\right]}^{*-1}(u) \leq H_{1\left[\xi_{1 q}\right]}^{*} \circ H_{1\left[\xi_{1} p\right]}^{*-1}(u),
$$

where $\xi_{i p}=F_{i}^{-1}(p)$ stands for the $p$ th quantile of the marginal distribution of $X_{i}$, and $H_{i[s]}^{*}$ denotes the conditional distribution of $Y_{i}$ given $X_{i}>s$, for $i=1,2$.

It is easy to see that both more SI order and more RTI order are copula-based orders, and more SI order implies more RTI order which in turn implies more concordance ordering (i.e., the two copulas are ordered). For the concept of copula, please refer to Nelsen [59] for more details.

As observed in Avérous et al. [57] and Genest et al. [60], there is a close connection between the above concepts of more dependence and the notion of dispersive ordering.

\subsection{Notions of Majorization and Related Orderings}

One of the basic tools in establishing various inequalities in statistics and probability is the notion of majorization.

Let $\left\{x_{(1)} \leq x_{(2)} \leq \cdots \leq x_{(n)}\right\}$ denote the increasing arrangement of the components of the vector $\mathbf{x}=\left(x_{1}, x_{2}, \ldots, x_{n}\right)$.

Definition 2.20. The vector $\mathbf{x}$ is said to majorize the vector $\mathbf{y}$ (written $\mathbf{x} \geq \mathbf{m} \geq$ ) if $\sum_{i=1}^{j} x_{(i)} \leq$ $\sum_{i=1}^{j} y_{(i)}$ for $j=1, \ldots, n-1$ and $\sum_{i=1}^{n} x_{(i)}=\sum_{i=1}^{n} y_{(i)}$.

Functions that preserve the majorization ordering are called Schur-convex functions. The vector $\mathbf{x}$ is said to majorize the vector $\mathbf{y}$ weakly (written $\mathbf{x} \geq \mathbf{w}$ ) if $\sum_{i=1}^{j} x_{(i)} \leq \sum_{i=1}^{j} y_{(i)}$ for $j=1, \ldots, n$. Marshall et al. [34] provides extensive and comprehensive details on the theory of majorization and its applications in statistics. order.

Bon and Păltănea [24] have considered a preorder on $\mathbb{R}^{+n}$, which they call as a $p$-larger

Definition 2.21. A vector $\mathbf{x}$ in $\mathbb{R}^{+n}$ is said to be $p$-larger than another vector $\mathbf{y}$ also in $\mathbb{R}^{+n}$ (written $\mathbf{x} \geq \mathbf{p}$ ) if $\prod_{i=1}^{j} x_{(i)} \leq \prod_{i=1}^{j} y_{(i)}, j=1, \ldots, n$. 
Let $\log (\mathbf{x})$ denote the vector of logarithms of the coordinates of $\mathbf{x}$. It is easy to verify that

$$
\mathbf{x} \stackrel{p}{\geq} \mathbf{y} \Longleftrightarrow \log (\mathbf{x}) \stackrel{w}{\geq} \log (\mathbf{y})
$$

It is known that $\mathbf{x} \stackrel{m}{\geq} \mathbf{y} \Rightarrow\left(g\left(x_{1}\right), \ldots, g\left(x_{n}\right)\right) \stackrel{w}{\geq}\left(g\left(y_{1}\right), \ldots, g\left(y_{n}\right)\right)$ for all concave functions $g$ (cf. [34]). From this and (2.33), it follows that when $\mathbf{x}, \mathbf{y} \in \mathbb{R}^{+n}$

$$
\mathbf{x} \stackrel{m}{\geq} \mathbf{y} \Longrightarrow \mathbf{x} \stackrel{p}{\geq} \mathbf{y}
$$

The converse is, however, not true. For example, the vectors $(0.2,1,5) \stackrel{p}{\geq}(1,2,3)$ but majorization does not hold between these two vectors.

\section{Stochastic Comparisons of Order Statistics in the One-Sample Problem}

In this section, we compare order statistics from a single sample according to various stochastic orders.

\subsection{Magnitude Orderings between Order Statistics in the One-Sample Problem}

Let $X_{1}, \ldots, X_{n}$ be a set of independent and identically distributed random variables. It is easy to see that $X_{i: n} \leq_{\mathrm{st}} X_{j: n}$, for all $1 \leq i<j \leq n$. Boland et al. [18] extended this result from usual stochastic order to hazard rate order. Using the definition of likelihood ratio ordering, it is not hard to prove that $X_{i: n} \leq_{\mathrm{r}} X_{j: n}$ for $1 \leq i<j \leq n$. Shaked and Shanthikumar [2] considered the problem of comparing order statistics from samples with possibly unequal sample sizes. They showed that if random variables $X_{i}^{\prime}$ s are iid, then $X_{n: n} \leq_{\operatorname{lr}} X_{n+1: n+1}$ and $X_{1: n} \geq_{\operatorname{lr}} X_{1: n+1}$. Raqab and Amin [61] strengthened this result and proved that $X_{i: n} \leq_{\operatorname{lr}} X_{j: m}$, whenever $i \leq j$ and $n-i \geq m-j$.

It is interesting to investigate the above stochastic relations among order statistics when the random variables are independent but not identically distributed. Boland et al. [18] showed that if random variables are independent and $X_{k} \leq_{\mathrm{hr}} X_{n+1}, k=1, \ldots, n$, then $X_{i-1: n} \leq_{\mathrm{hr}} X_{i: n+1}, i=1, \ldots, n+1$. They also proved that if $X_{i}^{\prime}$ 's are independent and $X_{n+1} \leq_{\mathrm{hr}} X_{k}$, $k=2, \ldots, n$, then $X_{i: n} \geq_{\mathrm{hr}} X_{i: n+1}, i=1, \ldots, n$. Assuming $X_{1} \leq_{\mathrm{lr}} X_{2} \leq_{\mathrm{lr}} \cdots \leq_{\mathrm{lr}} X_{n}$, Bapat an Kochar [17] proved that $X_{i: n} \leq_{\operatorname{lr}} X_{\mathrm{j}: n}, i<j$.

\subsection{Variability Orderings between Order Statistics in the One-Sample Problem}

David and Groeneveld [62] proved that if $X_{i}$ 's are iid random variables with a common decreasing failure rate (DFR) distribution, then $\operatorname{var}\left(X_{i: n}\right) \leq \operatorname{var}\left(X_{j: n}\right)$, for $1 \leq i<j \leq n$. Kochar [11] strengthened this result to prove that under the same conditions, $X_{i: n} \leq \operatorname{disp} X_{j: n}$, 
$1 \leq i<j \leq n$. Khaledi and Kochar [27] further proved that if $X_{i}{ }^{\prime}$ s are iid with a DFR distribution, then $X_{i: n} \leq_{\operatorname{disp}} X_{j: m}$, whenever $i \leq j$ and $n-i \geq m-j$.

\subsection{Skewness Orderings between Order Statistics in the One-Sample Problem}

Arnold and Villasenor [63], Arnold and Nagaraja [64], Wilfling [65], and Kleiber [66], among others, studied Lorenz order relations between order statistics from uniform and other distributions. In particular, Arnold and Villasenor [63] proved the following result on Lorenz ordering between the order statistics from uniform distributions.

Theorem 3.1. Let $U_{i: n}$ denote the ith order statistic of a random sample of size $n$ from a uniform distribution over $(0,1), i=1, \ldots, n$. Then

(a) $U_{i+1: n} \leq_{\text {Lorenz }} U_{i: n}$, for all $i \leq n-1$,

(b) $U_{i: n} \leq_{\text {Lorenz }} U_{i: n+1}$, for all $i \leq n+1$,

(c) $U_{n-i+1: n+1} \leq_{\text {Lorenz }} U_{n-i: n}$, for all $i \leq n$,

(d) $U_{n+2: 2 n+3} \leq_{\text {Lorenz }} U_{n+1: 2 n+1}$, for all $n$.

The last inequality may be described as "sample medians exhibit less variability as sample size increases." Arnold and Villasenor [63] wonder about the conditions on $i, j, m$, and $n$ under which

$$
U_{j: m} \leq_{\text {Lorenz }} U_{i: n}
$$

holds.

Kochar [67] answered this question in Theorem 3.2 below in which sufficient conditions on the parent distribution $F$ are obtained under which $X_{j: m} \leq_{\star} X_{i: n}$ holds. Many of the previously known results follow from this general result as particular cases as star ordering implies Lorenz ordering.

Theorem 3.2. For $i=1, \ldots, n$, let $X_{i: n}$ denote the $i$ th order statistic of a random sample of size $n$ from a distribution with reverse hazard rate $\tilde{r}_{F}$. If

$$
x \tilde{r}_{F}(x) \text { is increasing in } x \text {, }
$$

then for $i \leq j$ and $n-i \geq m-j$,

$$
X_{j: m} \leq_{\star} X_{i: n}
$$

Remark 3.3. Arnold and Villasenor [63] mention (3.2) as a sufficient condition for the relation

$$
X_{i+1: n} \leq_{\text {Lorenz }} X_{i: n}
$$

to hold. We have a more general result. 
The following theorem is also proved in in Kochar [67].

Theorem 3.4. For $i=1, \ldots, n$, let $X_{i: n}$ denote the $i$ th order statistic of a random sample of size $n$ from a distribution with its hazard rate $r_{F}(x)=f(x) / \bar{F}(x)$ satisfying the condition,

$$
x r_{F}(x) \text { is decreasing in } x .
$$

Then

$$
X_{i: n} \leq_{\star} X_{j: m} \text { for } i \leq j, n-i \geq m-j .
$$

The above theorems immediately lead to the following result because of the relation between star ordering and Lorenz ordering.

Corollary 3.5. If for $i=1, \ldots, n, X_{i: n}$ denotes the ith order statistic of a random sample of size $n$ from a distribution satisfying

(a) condition (3.2), then

$$
X_{j: m} \leq_{\text {Lorenz }} X_{i: n}
$$

for $i \leq j$ and $n-i \geq m-j$.

(b) condition (3.5), then

$$
X_{j: m} \geq_{\text {Lorenz }} X_{i: n}
$$

$$
\text { for } i \leq j \text { and } n-i \geq m-j \text {. }
$$

Example 3.6. The condition (3.2) is satisfied by the power function distribution with distribution function, $F_{X}(x)=[x / c]^{\gamma}, o \leq x \leq c, \gamma>0$. Therefore, the conclusions of Theorem 3.2 and Corollary 3.5(a) hold for this distribution. Arnold and Villasenor [63] also conjectured that for this distribution,

$$
X_{n-j+1: n+1} \leq_{\text {Lorenz }} X_{n-j: n,}, \quad \text { for every } 1 \leq j \leq n .
$$

Its proof immediately follows from Theorem 3.2 and Corollary 3.5.

Example 3.7. Let $X$ has Pareto distribution with $F(x)=1-(x / c)^{-\alpha}, x>c, \alpha>1$. Condition (3.5) is satisfied by this distribution. Hence the conclusions of Theorem 3.4 and Corollary 3.5(b) hold for this distribution. 


\subsection{Dependence among Order Statistics in the One-Sample Problem}

Assuming that $X_{1}, \ldots, X_{n}$ are independent and identically random variables, Bickel [68] showed that

$$
\operatorname{Cov}\left(X_{i: n}, X_{j: n}\right) \geq 0
$$

When the parent distribution has an increasing hazard rate and a decreasing reverse hazard rate, Tukey [69] showed that

$$
\operatorname{Cov}\left(X_{i^{\prime}: n}, X_{j^{\prime}: n}\right) \leq \operatorname{Cov}\left(X_{i: n}, X_{j: n}\right)
$$

for either $i=i^{\prime}$ and $j \leq j^{\prime}$; or $j=j^{\prime}$ and $i^{\prime} \leq i$.

Kim and David [70] proved that if both the hazard and the reverse hazard rates of the $X_{i}$ 's are increasing, then inequality (3.11) remains valid when $i=i^{\prime}$ and $j \leq j^{\prime}$; However, the inequality (3.11) is reversed when $j=j^{\prime}$ and $i^{\prime} \leq i$.

Avérous et al. [57] used the more SI concept to study the relative degree of dependence between two pairs of random variables. They proved the following result.

Theorem 3.8. Let $X_{1: n} \leq \cdots X_{n: n}$ and $X_{1: n^{\prime}} \leq \cdots X_{n^{\prime}: n^{\prime}}$ be the order statistics associated with two independent random samples of sizes $n$ and $n^{\prime}$ from the same continuous distribution. Then, for $1 \leq$ $i \leq j \leq n, 1 \leq i^{\prime} \leq j^{\prime} \leq n^{\prime}$, and $i^{\prime} \leq i, j-i \leq j^{\prime}-i^{\prime}, n-i \leq n^{\prime}-i^{\prime}, n^{\prime}-j^{\prime} \leq n-j$, one hold that

$$
\left(X_{j^{\prime}: n^{\prime}} \mid X_{i^{\prime}: n^{\prime}}\right) \preceq_{S I}\left(X_{j: n} \mid X_{i: n}\right)
$$

As a direct consequence, we have the following result.

Corollary 3.9. Let $X_{1: n} \leq \cdots X_{n: n}$ be order statistics from the same continuous distribution. Then,

(1) $\left(X_{k: n} \mid X_{i: n}\right) \leq_{\mathrm{SI}}\left(X_{j: n} \mid X_{i: n}\right)$ for $1 \leq i<j<k \leq n$;

(2) $\left(X_{j: n} \mid X_{i: n}\right) \leq \mathrm{sI}\left(X_{j+1: n+1} \mid X_{i+1: n+1}\right)$ for $1 \leq i<j \leq n$;

(3) $\left(X_{n+1: n+1} \mid X_{1: n+1}\right) \leq$ sI $\left(X_{n: n} \mid X_{1: n}\right)$ for $n \geq 2$.

It can be seen from the above result that the dependence between the components of a pair $\left(X_{j: n}, X_{i: n}\right)$ of order statistics decreases in the sense of SI ordering as $i$ and $j$ get further apart.

Remark 3.10. Since the copula of a pair of order statistics of a random sample is independent of the parent distribution and since the concept of more SI is copula based, it follows that in Theorem 3.8 and Corollary 3.9, the two samples could be from different distributions.

As explained in Avérous et al. [57], the following result follows immediately from Theorem 3.8.

Corollary 3.11. Under the assumptions of Theorem 3.8, one has

$$
\kappa\left(X_{j^{\prime}: n^{\prime}}, X_{i^{\prime}: n^{\prime}}\right) \leq \kappa\left(X_{j: n}, X_{i: n}\right)
$$


where $\kappa(X, Y)$ represents Spearman's rho, Kendall's tau, Gini's coefficient, or indeed any other copulabased measure of concordance satisfying the axioms of Scarsini [71].

\subsubsection{Kendall's $\tau$ and Spearman's $\rho$ for Order Statistics}

Two popular nonparametric measures of association for bivariate random variables are Kendall's $\tau$ and Spearman's $\rho$, which measure different aspects of the dependence structure. In terms of dependence properties, Spearman's $\rho$ is a measure of average quadrant dependence, while Kendall's $\tau$ is a measure of average likelihood ratio dependence (cf. $[59,72])$.

Avérous et al. [57] made an important observation that in the case of a random sample from a continuous distribution with cdf $F$, the copula of a pair of order statistics is independent of the parent distribution $F$. As a result the value of any copula-based measure of dependence like Kendall's tau or Spearman's coefficient for any pair of order statistics $\left(X_{i: n}, X_{j: n}\right)$ will be the same for all continuous distributions Schmitz [73] derived the following formulas:

$$
\begin{gathered}
\tau\left(X_{1: n}, X_{n: n}\right)=\frac{1}{2 n-1}, \\
\rho\left(X_{1: n}, X_{n: n}\right)=3-\frac{12 n}{\left(\begin{array}{c}
2 n \\
n
\end{array}\right)} \sum_{k=0}^{n} \frac{(-1)^{k}}{2 n-k}\left(\begin{array}{c}
2 n \\
n+k
\end{array}\right)+12 \frac{(n !)^{3}}{(3 n) !}(-1)^{n} .
\end{gathered}
$$

X. Li and Z. Li [74] proved a conjecture in Schmitz [73] that

$$
\frac{\rho\left(X_{1: n}, X_{n: n}\right)}{\tau\left(X_{1: n}, X_{n: n}\right)} \longrightarrow \frac{3}{2}, \quad n \longrightarrow \infty
$$

Avérous et al. [57] used a combinatorial approach to prove the following formula of Kendall's $\tau$ for any pair of order statistics from the same continuous distribution:

$$
\tau\left(X_{i: n}, X_{j: n}\right)=1-\frac{2(n-1)}{2 n-1}\left(\begin{array}{c}
n-2 \\
i-1
\end{array}\right)\left(\begin{array}{c}
n-i-1 \\
j-i-1
\end{array}\right) \sum_{s=0}^{n-j} \sum_{r=0}^{i-1} \frac{\left(\begin{array}{c}
n \\
r
\end{array}\right)\left(\begin{array}{c}
n-r \\
s
\end{array}\right)}{\left(\begin{array}{c}
2 n-2 \\
n-j+s, r+i-1
\end{array}\right)}
$$

Subsequently, Chen [75] developed three new formulas to compute $\rho\left(X_{1: n}, X_{n: n}\right)$ :

$$
\rho\left(X_{1: n}, X_{n: n}\right)=3\left(1-4 a_{n}\right)
$$

where $a_{n}$ can be computed by any one of the following formulas.

(1) Formula 1:

$$
a_{n}=n(n-1) \int_{0}^{1} \int_{0}^{t}(1-s)^{n} t^{n}(t-s)^{n-2} d s d t
$$


(2) Formula 2:

$$
a_{n}=\sum_{k=0}^{n}(-1)^{k} \frac{\left(\begin{array}{c}
n \\
k
\end{array}\right)}{\left(\begin{array}{c}
n+k \\
k
\end{array}\right)} \frac{n+k}{2 n+k}
$$

(3) Formula 3:

$$
a_{n}=\frac{n(n-1)}{(3 n) !} \sum_{j=0}^{n} \frac{n !}{j !} \sum_{k=0}^{n} \frac{n !}{k !}(n-2+j+k) !
$$

He further showed the following compound inequality:

$$
\frac{3(2 n-1)\left(14 n^{2}+15 n+3\right)}{56 n^{3}+86 n^{2}+43 n+7} \leq \frac{\rho\left(X_{1: n}, X_{n: n}\right)}{\tau\left(X_{1: n}, X_{n: n}\right)} \leq \frac{3(2 n-1)\left(14 n^{2}-13 n+2\right)}{56 n^{3}-82 n^{2}+39 n-6}
$$

from which, (3.15) follows immediately.

Recently, Navarro and Balakrishnan [76] have also studied this problem and have obtained alternate expressions for computing these measures of dependence.

\subsubsection{Dependence Orderings among Order Statistics When the Observations Are Not Identically Distributed}

The proportional hazard rates (PHRs) model is a popular model in survival analysis, reliability theory, and other branches of statistics. Let $X_{i}$ denote the lifetime of the ith component of a reliability system with survival function $\bar{F}_{i}(t), i=1, \ldots, n$. Then they have proportional hazard rates if there exist constants $\lambda_{1}, \ldots, \lambda_{n}$ and a (cumulative hazard) function $R(t) \geq 0$ such that $\bar{F}_{i}(t)=e^{-\lambda_{i} R(t)}$ for $i=1, \ldots, n$. Clearly then the hazard rate of $X_{i}$ is $r_{i}(t)=\lambda_{i} R^{\prime}(t)$ (assuming it exists). An example of such a situation is when the components have independent exponential lifetimes with respective hazard rates $\lambda_{1}, \ldots, \lambda_{n}$.

Boland et al. [10] studied in detail the dependence properties of order statistics when the observations are not necessarily identically distributed. They proved the following dependence result for the PHR model.

Theorem 3.12. Let $X_{1}, \ldots, X_{n}$ be independent random variables with differentiable densities and follow the PHR model on an interval. Then for $1<i \leq n, X_{i: n}$ is SI in $X_{1: n}$.

They also gave a counterexample to illustrate that, in general, $X_{i: n}$ is not SI in $X_{1: n}$. However, they showed that for $1 \leq i<j \leq n, X_{j: n}$ is RTI in $X_{i: n}$.

Theorem 3.13. Let $X_{1}, \ldots, X_{n}$ be independent random variables. Then for any $i \leq j, R T I\left(X_{j: n} \mid X_{i: n}\right)$ and $\operatorname{LTD}\left(X_{i: n} \mid X_{j: n}\right)$.

They showed with the help of a counter example that, in general, the relation $\operatorname{RTI}\left(X_{i: n} \mid\right.$ $X_{j: n}$ ) may not hold for $i<j$. 
This topic has been further developed by $\mathrm{Hu}$ and Xie [77], where they exploited the negative dependence of occupancy numbers in the balls and bins experiment. They proved the following result.

Theorem 3.14. Let $X_{1}, \ldots, X_{n}$ be independent random variables. For $1 \leq i \leq j_{1} \leq j_{2} \leq \cdots \leq j_{r} \leq n$, and $\left(x_{1}, \ldots, x_{n}\right) \in \mathbb{R}^{n}$,

(1) if $A_{i, n, y}=\left\{X_{i: n}>y\right\}$, then

$$
P\left(X_{j_{1}}>x_{1}, \ldots, X_{j_{r}}>x_{r} \mid A_{i, n, y}\right)
$$

is increasing in $y$;

(2) if event $A_{i, n, y}$ is either $\left\{X_{i: n}>y\right\}$ or $\left\{X_{i: n} \leq y\right\}$, then the conditional probability in (3.22) is decreasing in $i$ for each $y$; and

(3) if each $X_{k}$ has a continuous distribution function, and if $A_{i, n, y}$ is either $\left\{X_{i: n}=y\right\}$ or $\left\{X_{i-1: n}<y<X_{i: n}\right\}$, then (3.22) is decreasing in i for each $y$, where $X_{0: n} \equiv-\infty$.

Dubhashi and Häggström [78] further extended the above result to multivariate stochastic comparisons.

Theorem 3.15. Let $X_{1}, \ldots, X_{n}$ be independent random variables. Then

$$
\left[\left(X_{i: n}, \ldots, X_{n: n}\right) \mid X_{i: n}>y\right] \preceq_{\text {st }}\left[\left(X_{i: n}, \ldots, X_{n: n}\right) \mid X_{i: n}>y^{\prime}\right], \quad y \leq y^{\prime} .
$$

Subsequently, Theorem 3.14 was further extended by Hu and Chen [79] as follows.

Theorem 3.16. Let $X_{1}, \ldots, X_{n}$ be independent random variables.

(1) If $j-i \geq \max \{n-m, 0\}$, then

$$
P\left(X_{j: n}>x_{1}, X_{j+1: n}>x_{2}, \ldots, X_{n: n}>x_{n-j+1} \mid X_{i: m}>y\right)
$$

is increasing in $y$ for all $\left(x_{1}, \ldots, x_{n-j+1}\right) \in \mathbb{R}^{n-j+1}$;

(2) If $j-i \leq \min \{n-m, 0\}$, then

$$
P\left(X_{1: n} \leq x_{1}, X_{2: n} \leq x_{2}, \ldots, X_{j: n} \leq x_{n-j+1} \mid X_{i: m} \leq y\right)
$$

is decreasing in $y$ for all $\left(x_{1}, \ldots, x_{j}\right) \in \mathbb{R}^{j}$.

Recently, Zhuang et al. [80] discussed the dependence among order statistics in the sense of multivariate stochastic comparisons, which extends the results in $\mathrm{Hu}$ and Chen [79] and Dubhashi and Häggström [78].

Theorem 3.17. Let $X_{1}, \ldots, X_{n}$ be independent random variables.

(1) If $j-i \geq \max \{n-m, 0\}$, then

$$
\left[\left(X_{j: n}, \ldots, X_{n: n}\right) \mid X_{i: m}>y\right] \preceq_{s t}\left[\left(X_{j: n}, \ldots, X_{n: n}\right) \mid X_{i: m}>y^{\prime}\right], \quad y \leq y^{\prime} .
$$


(2) If $j-i \leq \min \{n-m, 0\}$, then

$$
\left[\left(X_{1: n}, \ldots, X_{j: n}\right) \mid X_{i: m} \leq y\right] \preceq_{s t}\left[\left(X_{1: n}, \ldots, X_{j: n}\right) \mid X_{i: n} \leq y^{\prime}\right], \quad y \leq y^{\prime}
$$

\section{Stochastic Comparisons of Order Statistics in the Two-Sample Problem}

Let $X_{1}, \ldots, X_{n}$ be a set of independent random variables and $Y_{1}, \ldots, Y_{n}$ be another set of independent random variables. Ross [31] proved that if $X_{i} \leq_{\mathrm{st}} Y_{i}, i=1, \ldots, n$, then $\left(X_{1}, \ldots, X_{n}\right) \leq_{\mathrm{st}}\left(Y_{1}, \ldots, Y_{n}\right)$. A consequence of this result is that $X_{i: n} \leq_{\mathrm{st}} Y_{i: n}$ for $i=1, \ldots, n$. Lynch et al. [81] generalized this result from stochastic ordering to hazard rate ordering though under a stronger condition. They proved that if $X_{i} \leq_{h r} Y_{j}, i, j \in\{1, \ldots, n\}$, then $X_{i: n} \leq_{h r} Y_{i: n}, i=1, \ldots, n$. A similar result for likelihood ratio ordering has been proved by Chan et al. [82]. They proved that if $X_{i} \leq_{\operatorname{lr}} Y_{j}, i, j \in\{1, \ldots, n\}$, then $X_{i: n} \leq_{\operatorname{lr}} Y_{i: n}, i=1, \ldots, n$. Lillo et al. [83] in the following theorem strengthened this result to the case when the number of $X_{i}{ }^{\prime}$ s and $Y_{i}{ }^{\prime}$ s is not necessarily equal.

Theorem 4.1. Let $X_{1}, \ldots, X_{n}$ be independent random variables and let $Y_{1}, \ldots, Y_{m}$ be another set of independent random variables, all having absolutely continuous distributions. Then $X_{i} \leq l r Y_{j}$ for all $i, j$ implies $X_{i: n} \leq_{l r} Y_{j: m}$ whenever $i \leq j$ and $n-i \geq m-j$.

In the next theorem we establish dispersive ordering between order statistics when the random samples are drawn from different distributions.

Theorem 4.2. Let $X_{1}, \ldots, X_{n}$ be a random sample of size $n$ from a continuous distribution $F$ and let $Y_{1} \ldots, Y_{m}$ be a random sample of size $m$ from another continuous distribution $G$. If either $F$ or $G$ is $D F R$, then

$$
X \leq_{\text {disp }} Y \Longrightarrow X_{i: n} \leq_{\text {disp }} Y_{j: m} \text { for } i \leq j, n-i \geq m-j .
$$

Proof. Let $F$ be a DFR distribution. The proof for the case when $G$ is DFR is similar. Khaledi and Kochar [27] proved that $X_{i: n} \leq_{\operatorname{disp}} X_{j: m}$ for $i \leq j$ and $n-i \geq m-j$. Bartoszewicz [42] proved that if $X \leq_{\text {disp }} Y$ then $X_{j: m} \leq_{\text {disp }} Y_{j: m}$. Combining these we get the required result.

Since the property $X \leq_{\mathrm{hr}} Y$ together with the condition that either $F$ or $G$ is DFR implies that $X \leq_{\text {disp }} Y$, we get the following result from the above theorem.

Corollary 4.3. Let $X_{1}, \ldots, X_{n}$ be a random sample of size $n$ from a continuous distribution $F$ and $Y_{1} \ldots, Y_{m}$ be a random sample of size $m$ from another continuous distribution $G$. If either $F$ or $G$ is DFR, then

$$
X \leq_{h r} Y \Longrightarrow X_{i: n} \leq_{\text {disp }} Y_{j: m} \text { for } i \leq j, n-i \geq m-j .
$$




\subsection{Stochastic Comparisons of Order Statistics When Observations Are Heterogeneous}

Many researchers have investigated the effect on the survival function, the hazard rate function and other characteristics of the time to failure of this system when we switch the vector $\left(\lambda_{1}, \ldots, \lambda_{n}\right)$ to another vector say $\left(\lambda_{1}^{*}, \ldots, \lambda_{n}^{*}\right)$ in the PHR model. Pledger and Proschan [15], for the first time, studied this problem and proved the following interesting result among many other results.

Theorem 4.4. Let $\left(X_{1}, \ldots, X_{n}\right)$ and $\left(X_{1}^{*}, \ldots, X_{n}^{*}\right)$ be two random vectors of independent lifetimes with proportional hazards with $\lambda_{1}, \ldots, \lambda_{n}$ and $\lambda_{1}^{*}, \ldots \lambda_{n}^{*}$ as the constants of proportionality. Then for $i=1, \ldots, n$.

$$
\left(\lambda_{1}, \lambda_{2}, \ldots, \lambda_{n}\right) \stackrel{m}{\geq}\left(\lambda_{1}^{*}, \lambda_{2}^{*}, \ldots, \lambda_{n}^{*}\right) \Longrightarrow X_{k: n} \geq_{s t} X_{k: n}^{*} \text { for } k=1, \ldots, n
$$

Proschan and Sethuraman [16] strengthened this result from component wise stochastic ordering to multivariate stochastic ordering. That is, under the assumptions of Theorem 4.4, they proved that

$$
\left(X_{1: n}, \ldots, X_{n: n}\right) \geq_{s t}\left(X_{1: n}^{*}, \ldots, X_{n: n}^{*}\right)
$$

These results show that if the parent observations have proportional hazard rates, then their order statistics are stochastically larger when the proportionality parameters are dispersed in the sense of majorization.

Boland et al. [18] showed with the help of the following counterexample that (4.3) can not be strengthened from stochastic ordering to hazard rate ordering for $k=n$ when $n \geq 3$.

Example 4.5. Let $\left(X_{1}, X_{2}, X_{3}\right)$ be independent exponential random vector with hazard rate vector $\left(\lambda_{1}, \lambda_{2}, \lambda_{3}\right)=(0.1,1,9)$ and $\left(X_{1}^{*}, X_{2}^{*}, X_{3}^{*}\right)$ be independent exponential random vector with hazard rate vector $\left(\lambda_{1}^{*}, \lambda_{2}^{*}, \lambda_{3}^{*}\right)=(0.1,4,6)$. It is easily seen that

$$
\left(\lambda_{1}, \lambda_{2}, \lambda_{3}\right) \stackrel{m}{\geq}\left(\lambda_{1}^{*}, \lambda_{2}^{*}, \lambda_{3}^{*}\right)
$$

However,

$$
r_{X_{3: 3}}(2) \approx 0.113>r_{X_{3: 3}^{*}}(2) \approx 0.100 \text {. }
$$

Hence,

$$
X_{3: 3} \searrow_{\mathrm{hr}} X_{3: 3}^{*} \text {. }
$$

Khaledi and Kochar [28] proved the following result for the largest order statistics under $p$-larger ordering. 
Theorem 4.6. Let $\left(X_{1}, \ldots, X_{n}\right)$ and $\left(X_{1}^{*}, \ldots, X_{n}^{*}\right)$ be two random vectors of independent lifetimes with proportional hazards with $\lambda_{1}, \ldots, \lambda_{n}$ and $\lambda_{1}^{*}, \ldots \lambda_{n}^{*}$ as the constants of proportionality. Then

$$
\left(\lambda_{1}, \lambda_{2}, \ldots, \lambda_{n}\right) \stackrel{p}{\geq}\left(\lambda_{1}^{*}, \lambda_{2}^{*}, \ldots, \lambda_{n}^{*}\right) \Longrightarrow X_{n: n} \geq_{s t} X_{n: n}^{*}
$$

The following example due to Khaledi and Kochar [28] shows that Theorem 4.6 may not hold for other order statistics.

Example 4.7. Let $\left(X_{1}, X_{2}, X_{3}\right)$ be independent exponential random vector with hazard rate vector $\left(\lambda_{1}, \lambda_{2}, \lambda_{3}\right)=(0.1,1,7.9)$ and $\left(X_{1}^{*}, X_{2}^{*}, X_{3}^{*}\right)$ be independent exponential random vector with hazard rate vector $\left(\lambda_{1}^{*}, \lambda_{2}^{*}, \lambda_{3}^{*}\right)=(1,2,5)$. It is easily seen that

$$
\left(\lambda_{1}, \lambda_{2}, \lambda_{3}\right) \stackrel{p}{\geq}\left(\lambda_{1}^{*}, \lambda_{2}^{*}, \lambda_{3}^{*}\right)
$$

However,

$$
r_{X_{1: 3}}(x)=9>r_{X_{1: 3}^{*}}(x)=8
$$

which implies

$$
X_{1: 3} \leq_{\text {st }} X_{1: 3}^{*}
$$

Khaledi and Kochar [28] also showed that in, general,

$$
\left(\lambda_{1}, \lambda_{2}, \ldots, \lambda_{n}\right) \stackrel{p}{\geq}\left(\lambda_{1}^{*}, \lambda_{2}^{*}, \ldots, \lambda_{n}^{*}\right) \Rightarrow X_{n: n}^{*} \leq_{\text {rh }} X_{n: n}
$$

Torrado and Veerman [84] formulated a simple criteria in terms of the $\lambda_{i}$ 's to see whether two $k$-out-of- $n$ systems are stochastically ordered.

\subsubsection{Comparison of Order Statistics from Heterogenous and Homogeneous Samples.}

Let $X_{1}, \ldots, X_{n}$ be continuous independent random variables such that the c.d.f. of $X_{i}$ is $F_{i}$, $i=1, \ldots, n$, where $F_{i}$ 's are not necessarily all equal. Let $Y_{1}, \ldots, Y_{n}$ be a random sample of size $n$ from a distribution with c.d.f $G=(1 / n) \sum_{i=1}^{n} F_{i}$. Sen [7] proved a very general result that whereas for the first order statistic, $X_{1: n} \leq_{s t} Y_{1: n}$, the inequality is reversed for the largest order statistic, that is, $X_{n: n} \geq_{s t} Y_{n: n}$.

Dykstra et al. [22] studied the above problem for the exponential distributions. They proved the following stronger result on hazard rate ordering for largest order statistics. 
Theorem 4.8. Let $X_{1}, \ldots, X_{n}$ be independent exponential random variables with $X_{i}$ having hazard rate $\lambda_{i}, i=1, \ldots, n$; and let $Y_{1}, \ldots, Y_{n}$ be a random sample of size $n$ from an exponential distribution with common hazard rate $\lambda$. Then for

$$
\begin{gathered}
\lambda=\bar{\lambda}=\frac{\sum_{i=1}^{n} \lambda_{i}}{n}, \\
Y_{n: n} \leq h r X_{n: n}
\end{gathered}
$$

as well as

$$
Y_{n: n} \leq_{\text {disp }} X_{n: n} .
$$

Khaledi and Kochar [28] proved that (4.14) and (4.15) continue to hold with $\lambda=\widetilde{\lambda}=$ $\left(\prod_{i=1}^{n} \lambda_{i}\right)^{1 / n}$, the geometric mean of the $\lambda^{\prime} \mathrm{s}$. This result gives better bounds on the hazard rate and measures of variability of a parallel system with heterogeneous exponential components.

Khaledi and Kochar [28] also extended the results (4.14) and (4.15) from the exponential case to the PHR model as stated in the next theorem.

Theorem 4.9. Let $X_{1}, \ldots, X_{n}$ be independent random variables with $X_{i}$ having survival function $\bar{F}^{\lambda_{i}}, i=1, \ldots, n$. Let $Y_{1}, \ldots, Y_{n}$ be a random sample with common population survival distribution $\bar{F}^{\tilde{\lambda}}$, where $\tilde{\lambda}=\left(\prod_{i=1}^{n} \lambda_{i}\right)^{1 / n}$, then

(i) $Y_{n: n} \leq_{h r} X_{n: n ;}$

(ii) $Y_{n: n} \leq_{\text {disp }} X_{n: n}$ if $F$ has decreasing failure rate (DFR).

These results give nice bounds on the hazard rate and the variance of a parallel system with components which are independent following the PHR model in terms of the case when they are independent and identically distributed.

Kochar and $\mathrm{Xu}$ [85] further strengthened the conclusion (4.14) of Theorem 4.8 of Dykstra et al. [22] from hazard rate ordering to likelihood ratio order for the PHR model, which includes exponential as a special case.

Theorem 4.10. Let $X_{1}, \ldots, X_{n}$ be independent random variables with $X_{i}$ having survival function $\bar{F}^{\lambda_{i}}, i=1, \ldots, n$. Let $Y_{1}, \ldots, Y_{n}$ be a random sample with common population survival distribution $\bar{F}^{\bar{\lambda}}$, where $\bar{\lambda}=\sum_{i=1}^{n} \lambda_{i} / n$, then

$$
Y_{n: n} \leq_{l r} X_{n: n}
$$

The following example due to Kochar and $\mathrm{Xu}$ [85] shows that the result of Khaledi and Kochar [27] above cannot be strengthened from the hazard rate order to the likelihood ratio order under $p$-larger ordering.

Example 4.11. Let $X_{1}, \ldots, X_{n}$ be independent exponential random variables with $X_{i}$ having hazard rate $\lambda_{i}, i=1, \ldots, n$, and $Y_{1}, \ldots, Y_{n}$ be a random sample of size $n$ from an exponential 
distribution with common hazard rate $\tilde{\lambda}=\left(\prod_{i=1}^{n} \lambda_{i}\right)^{1 / n}$. Then, the reversed hazard rate of $X_{n: n}$ is

$$
\frac{f_{n: n}(x)}{F_{n: n}(x)}=\sum_{i=1}^{n} \frac{\lambda_{i} e^{-\lambda_{i} x}}{1-e^{-\lambda_{i} x}}
$$

Similarly, the reversed hazard rate of $Y_{n: n}$ is

$$
\frac{g_{n: n}(x)}{G_{n: n}(x)}=n \tilde{\jmath} \frac{e^{-\tilde{\lambda} x}}{1-e^{-\tilde{\lambda} x}}
$$

Let $\lambda_{1}=\lambda_{2}=1, \lambda_{3}=3$, and $n=3$, then,

$$
\frac{f_{n: n}(1)}{F_{n: n}(1)} \approx 1.321 \leq 1.339 \approx \frac{g_{n: n}(1)}{G_{n: n}(1)}
$$

Thus,

$$
X_{n: n} \nsupseteq_{\mathrm{rh}} Y_{n: n,}
$$

which implies that

$$
X_{n: n} \nsupseteq_{1 \mathrm{r}} Y_{n: n} .
$$

Bon and Păltănea [86] proved the following result on stochastic ordering between $k$ th order statistics from heterogeneous and homogeneous samples from exponential distributions.

Theorem 4.12. Let $X_{1}, \ldots, X_{p}$ be independent exponential random variables with hazard rates $\lambda_{i}$, $i=1, \ldots, n$. Let $Y_{1}, \ldots, Y_{n}$ be independent exponential random variables with a common hazard rate l. Then,

$$
\lambda \geq \hat{\jmath} \Longleftrightarrow X_{k: n} \geq_{s t} Y_{k: n}
$$

where

$$
\hat{\jmath}=\left(\left(\begin{array}{l}
n \\
k
\end{array}\right)^{-1} \sum_{1 \leq i_{1}<\cdots<i_{k} \leq n} \lambda_{i_{1}} \cdots \lambda_{i_{k}}\right)^{1 / k}
$$

a result which also follows from Khaledi and Kochar [27] for the case when $k=n$.

Kochar and $\mathrm{Xu}$ [87] proved the following results on excess wealth ordering between largest order statistics from heterogeneous and homogeneous samples. 
Theorem 4.13. Let $X_{1}, \ldots, X_{n}$ be independent exponential random variables with $X_{i}$ having hazard rate $\lambda_{i}, i=1, \ldots, n$. Let $Y_{1}, \ldots, Y_{n}$ be a random sample of size $n$ from an exponential distribution with common hazard rate $\lambda$. Then,

$$
\lambda^{*} \leq \lambda \Longleftrightarrow Y_{n: n} \leq_{R S} X_{n: n}
$$

where

$$
\lambda^{*}=\sum_{i=1}^{n} \frac{1}{i}\left[\sum_{k=1}^{n}(-1)^{k+1} \sum_{1 \leq i_{1} \leq \cdots \leq i_{k} \leq n} \frac{1}{\sum_{j=1}^{k} \lambda_{i_{j}}}\right]^{-1} .
$$

Theorem 4.14. Let $X_{1}, \ldots, X_{n}$ be independent random variables with $X_{i}$ having survival function $\bar{F}^{\lambda_{i}}, i=1, \ldots, n$, and let $Y_{1}, \ldots, Y_{n}$ be another random sample with the common survival function $\bar{F}^{\lambda}$. If $F$ is DFR, then

$$
\lambda^{*} \leq \lambda \Longrightarrow Y_{n: n} \leq_{R S} X_{n: n}
$$

where $\lambda^{*}$ is given in (4.24).

As a consequence, we have the following result.

Corollary 4.15. Let $X_{1}, \ldots, X_{n}$ be independent random variables with $X_{i}$ having survival function $\bar{F}^{\lambda_{i}}, i=1, \ldots, n$, and let $Y_{1}, \ldots, Y_{n}$ be another random sample with the common survival function $\bar{F}^{\lambda}$. If $F$ is $D F R$, then

$$
\lambda^{*} \leq \lambda \Longrightarrow \operatorname{Var}\left(Y_{n: n}\right) \leq \operatorname{Var}\left(X_{n: n}\right),
$$

where $\lambda^{*}$ is given in (4.24).

Remark 4.16. In the special case of exponential distribution, $\bar{F}(x)=e^{-x}$, Khaledi and Kochar [28] gave a lower bound on the variance of $X_{n: n}$ in terms of the geometric mean,

$$
\operatorname{Var}\left(X_{n: n}\right) \geq \frac{1}{\hat{\jmath}} \sum_{i=1}^{n} \frac{1}{i^{2}}
$$

Since the right spread order implies the variance being ordered (cf. Shaked and Shanthikumar [2]), Corollary 4.15 provides a better lower bound for the variance,

$$
\operatorname{Var}\left(X_{n: n}\right) \geq \frac{1}{\lambda^{*}} \sum_{i=1}^{n} \frac{1}{i^{2}}
$$

as $\lambda^{*} \leq \lambda$ 


\subsubsection{Skewness Comparisons of Order Statistics from Two Samples}

Kochar and $\mathrm{Xu}$ [87] for the first time studied the problem of comparing order statistics according to shape orderings like convex ordering and star ordering. They proved that a parallel system with heterogenous exponential component lifetimes is more skewed (according to convex transform order) than the one with i.i.d. exponential components. This is stated in the following theorem.

Theorem 4.17. Let $X_{1}, \ldots, X_{n}$ be independent exponential random variables with $X_{i}$ having hazard rate $\lambda_{i}, i=1, \ldots, n$. Let $Y_{1}, \ldots, Y_{n}$ be a random sample of size $n$ from an exponential distribution with common hazard rate $\lambda$. Then,

$$
Y_{n: n} \leq_{c} X_{n: n}
$$

Remark 4.18. Theorem 4.17 means that a parallel system with homogeneous exponential components ages faster than than a system with heterogenous exponential components in the sense of "more IFR" property. Note that a parallel system with homogeneous exponential components is IFR (Barlow and Proschan [49]). However, a parallel system with heterogenous exponential components may not be IFR.

Remark 4.19. It is interesting to note that, unlike the magnitude and variability orders, no restriction on the parameters is needed for Theorem 4.17 to hold as the convex transform order is scale invariant. Intuitively, due to the heterogeneity, the largest order statistic from a heterogenous sample will be more skewed than that from a homogeneous sample. Theorem 4.17 confirms this fact for exponential samples.

The following result, which is of independent interest in economics, is a direct consequence of Theorem 4.17

Corollary 4.20. Let $X_{1}, \ldots, X_{n}$ be independent exponential random variables with $X_{i}$ having hazard rate $\lambda_{i}, i=1, \ldots, n$. Let $Y_{1}, \ldots, Y_{n}$ be a random sample of size $n$ from an exponential distribution with common hazard rate $\lambda$. Then,

$$
Y_{n: n} \leq_{\text {Lorenz }} X_{n: n} .
$$

From Barlow and Proschan [49, page 60], it follows that,

$$
\begin{gathered}
E Y_{n: n}=\frac{1}{\lambda} \sum_{i=1}^{n} \frac{1}{i}, \\
\operatorname{Var}\left(Y_{n: n}\right)=\frac{1}{\lambda^{2}} \sum_{i=1}^{n} \frac{1}{i^{2}} .
\end{gathered}
$$

Using (2.25), one get the following lower bound on the coefficient of variation of $X_{n: n}$,

$$
\operatorname{cv}\left(X_{n: n}\right) \geq \frac{\left(\sqrt{\sum_{i=1}^{n}\left(1 / i^{2}\right)}\right)}{\left(\sum_{i=1}^{n}(1 / i)\right)} .
$$

Kochar and $\mathrm{Xu}$ [88] extended Theorem 4.17 to the PHR family as follows. 
Theorem 4.21. Let $X_{1}, \ldots, X_{n}$ be independent random variables with $X_{i}$ having survival function $\bar{F}^{\lambda_{i}}, i=1, \ldots, n$, and let $Y_{1}, \ldots, Y_{n}$ be a random sample from a distribution with the common survival distribution $\bar{F}^{\lambda}$ where $\lambda \geq \tilde{\lambda}=\sqrt[n]{\prod_{i=1}^{n} \lambda_{i}}$, the geometric mean of $\lambda_{i}$ 's. If

$$
\frac{R(x)}{x r(x)} \text { is increasing in } x \geq 0 \text {, }
$$

then

$$
X_{n: n} \geq_{\star} Y_{n: n}
$$

where $R(x)=-\log \bar{F}(x)$ is the cumulative hazard rate function, and $r(x)=f(x) / \bar{F}(x)$ is the hazard rate function of $F$.

It can be seen that distributions like Weibull, Lomax, and Pareto satisfy the condition (4.34). Kochar and $\mathrm{Xu}$ [88] proved the following result on star ordering for $k$ th order statistics.

Theorem 4.22. Let $X_{1}, \ldots, X_{p}$ be i.i.d. random variables with the common survival distribution $\bar{F}^{\lambda_{1}}$, and let $X_{p+1}, \ldots, X_{n}$ be another set of i.i.d. random variables with common survival distribution $\bar{F}^{\lambda_{2}}$, and let $Y_{1}, \ldots, Y_{n}$ be a random sample from a distribution with common survival distribution $\bar{F}^{\lambda}$ where $\lambda \geq \hat{\imath}$. If

$$
\frac{R(x)}{x r(x)} \text { is increasing in } x \geq 0
$$

then

$$
X_{k: n} \geq_{\star} Y_{k: n}
$$

and hence,

$$
X_{k: n} \geq_{\text {Lorenz }} Y_{k: n,}, k=1, \ldots, n,
$$

where

$$
\widehat{\jmath}=\left(\left(\begin{array}{l}
n \\
k
\end{array}\right)^{-1} \sum_{l \in \mathbb{L}}\left(\begin{array}{l}
p \\
l
\end{array}\right)\left(\begin{array}{c}
n-p \\
k-l
\end{array}\right) \lambda_{1}^{l} \lambda_{2}^{k-l}\right)^{1 / k}
$$

and $\mathbb{L}=\{l: \max \{k-n+p, 0\} \leq l \leq \min \{p, k\}\}$.

\subsubsection{Stochastic Comparisons of Second Order Statistics}

In the next theorem, we give a collection of results on various types of stochastic orders for the second order statistics. 
Theorem 4.23. Let $X_{1}, \ldots, X_{n}$ be independent exponential random variables with parameters $\lambda_{1}, \ldots, \lambda_{n}$, respectively; and let $Y_{1}, \ldots, Y_{n}$ be independent random variables with common hazard rate $\lambda$.

(a) (Păltănea [89])

$$
Y_{2: n} \leq_{h r} X_{2: n} \Longleftrightarrow \lambda \geq \hat{\imath}=\sqrt{\left(\begin{array}{l}
n \\
2
\end{array}\right)^{-1} \sum_{1 \leq i<j \leq n} \lambda_{i} \lambda_{j}}
$$

(b) (Zhao and Balakrishnan [90])

$$
Y_{2: n} \leq_{\text {disp }} X_{2: n} \Longleftrightarrow \lambda \geq \hat{\lambda}
$$

(c) (Zhao et al. [91])

$$
Y_{2: n} \leq_{l r} X_{2: n} \Longleftrightarrow \lambda \geq \lambda^{* *}=\frac{1}{2 n-1}\left(2 \Lambda_{1}+\frac{\Lambda_{3}-\Lambda_{1} \Lambda_{2}}{\Lambda_{1}^{2}-\Lambda_{2}}\right),
$$

where

$$
\Lambda_{k}=\sum_{i=1}^{k} \lambda_{i}^{k}, \quad k=1,2,3
$$

(d) (Zhao and Balakrishnan [92])

$$
\Upsilon_{2: n} \leq_{m r l} X_{2: n} \Longleftrightarrow \lambda \geq \lambda^{* *}
$$

where

$$
\lambda^{* *}=\frac{2 n-1}{n(n-1)\left[\sum_{i=1}^{n}\left(1 /\left(\Lambda_{1}-\lambda_{i}\right)-(n-1) / \Lambda_{1}\right)\right]}
$$

\subsection{The Scale Family}

Independent random variables $X_{\Lambda_{1}}, \ldots, X_{\lambda_{n}}$ are said to belong to the scale family of distributions if $X_{\lambda_{i}} \sim F\left(\lambda_{i} x\right)$, for $i=1, \ldots, n$, where $F$ is an absolutely continuous distribution function with density function $f$. It means that the random variables $\lambda_{1} X_{\lambda_{1}}, \ldots, \lambda_{n} X_{\lambda_{n}}$ are independent and identically distributed with common cdf $F$. $F$ is called the baseline distribution and the $\lambda_{i}{ }^{\prime}$ s are the scale parameters. It includes many important distributions like normal, exponential, Weibull, and gamma as special cases. The scale model is of theoretical as well as practical importance in various fields of probability and statistics. As we have seen above, there is an extensive literature on stochastic orderings among order statistics when the observations follow the exponential distribution with different scale parameters.

Pledger and Proschan [15] proved the following general result for the scale model. 
Theorem 4.24. Let $X_{1}, \ldots, X_{n} ; Y_{1}, \ldots, Y_{n}$ be independent nonnegative random variables with $X_{i} \sim$ $F\left(\lambda_{i} x\right), Y_{i} \sim F\left(\mu_{i} x\right), \lambda_{i}>0, \mu_{i}>0, i=1, \ldots, n$ where $F$ is an absolutely continuous distribution. If $r(x)$, the hazard rate of $F$, is decreasing, then

$$
\left(\lambda_{1}, \ldots, \lambda_{n}\right) \stackrel{m}{\geq}\left(\mu_{1}, \ldots, \mu_{n}\right) \Longrightarrow X_{k: n} \geq_{s t} Y_{k: n}
$$

for $k=1, \ldots, n$.

Bon and Păltănea [86] proved the following result for the scale model when one set of variables are iid.

Theorem 4.25. Let $X_{1}, \ldots, X_{n}$ be independent nonnegative random variables with $X_{i} \sim F\left(\lambda_{i} x\right)$ and let $Y_{1}, \ldots, Y_{n}$ be a random sample from a distribution with $c d f F\left(m_{k}(\lambda) x\right)$, where $F$ is the cdf of a nonnegative random variable with nonincreasing failure rate and

$$
m_{k}(\lambda)=\left(\left(\begin{array}{l}
n \\
k
\end{array}\right)^{-1} \sum_{1 \leq i_{1}<\cdots<i_{k} \leq n} \lambda_{i_{1}} \cdots \lambda_{i_{k}}\right)^{1 / k}
$$

If $F(x) /[x \bar{F}(x)]$ is an increasing function on $[0, \infty)$, then for $k \in\{1, \ldots, n\}$,

$$
X_{k: n} \geq_{s t} Y_{k: n}
$$

$\mathrm{Hu}$ [93] also considered the general scale problem and proved the following result.

Theorem 4.26. Let $X_{1}, \ldots, X_{n} ; Y_{1}, \ldots, Y_{n}$ be independent nonnegative random variables with $X_{i} \sim$ $F\left(\lambda_{i} x\right), Y_{i} \sim F\left(\mu_{i} x\right)$, where $\lambda_{i}>0, \mu_{i}>0, i=1, \ldots, n$ are such that

$$
\left(\lambda_{1}, \ldots, \lambda_{n}\right) \stackrel{m}{\geq}\left(\mu_{1}, \ldots, \mu_{n}\right)
$$

Assume that the failure rate of $F, r(x)$ is decreasing and $x r(x)$ is increasing. Then on some probability space $(\Omega, \mathcal{F}, D)$, there exist random variables $X_{1}^{\prime}, \ldots, X_{n}^{\prime} ; Y_{1}^{\prime}, \ldots, Y_{n}^{\prime}$ such that

$$
\begin{gathered}
\left(X_{1}, \ldots, X_{n}\right) \stackrel{s t}{=}\left(X_{1}^{\prime}, \ldots, X_{n}^{\prime}\right), \\
\left(Y_{1}, \ldots, Y_{n}\right) \stackrel{s t}{=}\left(Y_{1}^{\prime}, \ldots, Y_{n}^{\prime}\right), \\
X_{k: n} \geq Y_{k: n}, \quad \text { a.s. for } k=1, \ldots, n .
\end{gathered}
$$

This implies in particular that

$$
\left(X_{1: n}, \ldots, X_{n: n}\right) \geq\left(Y_{1: n}, \ldots, X_{n: n}\right)
$$

Let $G(\alpha, \lambda)$ and $W(\alpha, \lambda)$ denote gamma and Weibull random variables with shape parameter $\alpha$ and scale parameter $\lambda$. For these scale models, Sun and Zhang [94] and Khaledi and Kochar [95], respectively, proved the following theorem. 
Theorem 4.27. Let $\left(X_{1}, \ldots, X_{n}\right)$ and $\left(Y_{1}, \ldots, Y_{n}\right)$ be two sets of independent random variables with

(a) $X_{i} \sim W\left(\alpha, \lambda_{i}\right)$ and $Y_{i} \sim W\left(\alpha, \mu_{i}\right), i=1, \ldots, n$ or

(b) $X_{i} \sim G\left(\alpha, \lambda_{i}\right)$ and $Y_{i} \sim G\left(\alpha, \mu_{i}\right), i=1, \ldots, n$.

Then for $0<\alpha \leq 1$,

$$
\mathcal{H} \stackrel{m}{\geq} \boldsymbol{\mu} \Longrightarrow\left(X_{1: n}, \ldots, X_{n: n}\right) \stackrel{s t}{\geq}\left(Y_{1: n}, \ldots, Y_{n: n}\right)
$$

\subsubsection{Generalized Gamma Distribution}

A random variable $X$ is said to have a generalized gamma distribution, denoted by $X \sim$ $G G(p, q)$, if it admits the following density function:

$$
g_{p, q}(x)=\frac{p}{\Gamma(q / p)} x^{q-1} e^{-x^{p}} x>0
$$

where $p, q(>0)$ are the shapes parameters. It was introduced by Stacy [96] and includes the wildly used exponential $(p=1, q=1)$, Weibull $(q=p)$, and gamma $(p=1)$ distributions as special cases. It is a flexible family of distributions, having an increasing failure rate when $q \geq 1, p \geq 1$, a bathtub failure rate when $q<1, p>1$, an upside down bathtub (or unimodal) failure rate when $q>1, p<1$.

It is easy to show that for $p \leq 1, q \leq 1$, the hazard rate of generalized gamma distribution is decreasing. It is shown in Khaledi et al. [97] that $x r(x)$ is an increasing function of $x$ for all $p, q>0$. Thus the conditions of Theorem 4.26 are satisfied when the baseline distribution is generalized gamma distribution with $p \leq 1, q \leq 1$ and thus extending the result of Theorem 1.1 in Sun and Zhang [94] from standard gamma distribution to generalized gamma distribution.

\subsubsection{Power-Generalized Weibull Distribution}

A random variable $X$ is said to have power-generalized Weibull distribution, denoted by $X \sim \operatorname{PGW}(v, \gamma)$, if its density function is

$$
f(t, v, \gamma)=\frac{v}{\gamma} t^{\nu-1}\left(1+t^{\nu}\right)^{1 / \gamma-1} e^{1-\left(1+t^{\nu}\right)^{1 / \gamma}}, \quad t>0 ; v, \gamma>0
$$

and its survival function is

$$
\bar{F}(t, v, \gamma)=e^{1-\left(1+t^{\nu}\right)^{1 / \gamma}}, \quad t>0
$$

It has a decreasing failure rate when $v \leq \gamma, v \leq 1$, an increasing failure rate when $v \geq \gamma, v \geq 1$, a bathtub failure rate when $0<\gamma<v<1$, and an upside down bathtub (or unimodal) failure rate when $r>v>1$. It includes Weibull and exponential distributions as special cases.

For more details on this family and its applications in probability and statistics, the reader is referred to Bagdonavicius and Nikulin [98]. Let $r(x)$ be the hazard rate function 
of power-generalized Weibull distribution. It is known that for $v \leq r, 0<v \leq 1, r(x)$ is a decreasing function (cf. [98]).

For all values of $v$ and $\gamma$, the function

$$
x r(x)=\frac{v}{r} x^{v}\left(1+x^{v}\right)^{1 / \gamma-1}
$$

is increasing in $x$. That is, the conditions of Theorem 4.26 are satisfied by the powergeneralized Weibull distribution with $v \leq \gamma, 0<v \leq 1$, thus extending the result of Theorem 2.2 in Khaledi and Kochar [95] from standard Weibull distribution to power-generalized Weibull distribution.

Khaledi et al. [97] proved that under some additional conditions the results of Theorem 4.24 can be extended from stochastic ordering to the hazard rate order when we compare two series systems.

Theorem 4.28. Let $X_{\lambda_{1}}, \ldots, X_{\lambda_{n}}$ be independent nonnegative random variables with $X_{\lambda_{i}} \sim F\left(\lambda_{i} x\right)$, $i=1, \ldots, n$, where $\lambda_{i}>0, i=1, \ldots, n$ and $F$ is an absolutely continuous distribution. Let $r$ be the hazard rate functions of $F$. If $x^{2} r^{\prime}(x)$ is decreasing and $\left(\lambda_{1}, \ldots, \lambda_{n}\right) \geq\left(\mu_{1}, \ldots, \mu_{n}\right)$, then

(i) $X_{1: n} \geq h r Y_{1: n}$, and

(ii) if $r(x)$ is decreasing then $X_{1: n} \geq_{\text {disp }} Y_{1: n}$.

The inequalities in (i) and (ii) are reversed if $x^{2} r^{\prime}(x)$ is increasing.

It is shown in Khaledi et al. [97] that $x^{2} r^{\prime}(x)$ is decreasing for the $\operatorname{PGW}(v, r)$ distribution when $0<v \leq \gamma$ and $v<1$. This is also true for the $G G(p, q)$ distribution when $p, q<1$. We know that for $p, q \leq 1$, the hazard rate function $r(x)$ is decreasing. That is, Theorem 4.28 (ii) can be applied to this case as well.

Khaledi et al. [97] also established a stochastic ordering result for the largest order statistics under $p$-larger ordering.

Theorem 4.29. Let $X_{1}, \ldots, X_{n}$ be a set of independent nonnegative random variables with $X_{i} \sim$ $F\left(\lambda_{i} x\right), i=1, \ldots, n$, where $F$ is an absolutely continuous distribution function with density function $f$. Let $Y_{1}, \ldots, Y_{n}$ be another set of independent nonnegative random variables with $Y_{i} \sim F\left(\mu_{i} x\right)$, $i=1, \ldots, n$. Let $\tilde{r}(x)$ denote the reverse hazard rate of $F$. If $x \tilde{r}(x)$ is decreasing in $x$, then

$$
\left(\lambda_{1}, \ldots, \lambda_{n}\right) \stackrel{p}{\geq}\left(\mu_{1}, \ldots, \mu_{n}\right) \Longrightarrow X_{n: n} \geq_{s t} Y_{n: n}
$$

The above theorem immediately leads to the following corollary.

Corollary 4.30. Let $X_{1}, \ldots, X_{n}$ be a set of independent nonnegative random variables with $X_{i} \sim$ $F\left(\lambda_{i} x\right), i=1, \ldots, n$, where $F$ is an absolutely continuous distribution function with density function $f$. Let $Y_{1}, \ldots, Y_{n}$ be i.i.d. random variables with common c.d.f $F(\tilde{\lambda} x)$, where $\tilde{\lambda}$ is the geometric mean of the $\lambda_{i}$ 's. If $x \tilde{r}(x)$ is decreasing in $x$, then $X_{n: n} \geq_{s t} Y_{n: n}$.

The above corollary gives a lower bound on the survival function of a parallel system with non-identical components in terms of the one with i.i.d. components when the common scale parameter is the geometric mean of the scale parameters. The new bound is better than 
the one that follows from $\mathrm{Hu}$ [93] which is in terms of the arithmetic mean of the scale parameters since $\bar{F}_{X_{n: n}}(x)$ is a nonincreasing function of $\tilde{\lambda}$ and the fact that the geometric mean of the $\lambda_{i}$ 's is smaller than their arithmetic mean.

Under the conditions of Theorems 4.28 and 4.29 the improvements on the bounds are relatively more if the $\lambda_{i}$ 's are more dispersed in the sense of majorization. This fact follows from the fact that the geometric mean is Schur concave whereas the arithmetic mean is Schur constant and the survival function of a parallel system of i.i.d components with baseline distribution $F(x)$ and common parameter $\tilde{\lambda}$ is decreasing in $\tilde{\lambda}$.

It is shown in Khaledi et al. [97] that the conditions of Theorem 4.29 are satisfied when the baseline distributions in the scale model are generalized gamma distribution and powergeneralized Weibull distribution with arbitrary parameters.

The next theorem again by Khaledi et al. [97] extends Theorem 4.26 from stochastic ordering to the reverse hazard rate order when we compare two parallel systems, a result which is similar to Theorem 4.28.

Theorem 4.31. Let $X_{\lambda_{1}}, \ldots, X_{\lambda_{n}}$ be independent nonnegative random variables with $X_{\lambda_{i}} \sim F\left(\lambda_{i} x\right)$, $i=1, \ldots, n$, where $\lambda_{i}>0, i=1, \ldots, n$ and $F$ is an absolutely continuous distribution. Let $Y_{1}, \ldots, Y_{n}$ be another set of independent nonnegative random variables with $Y_{i} \sim F\left(\mu_{i} x\right), i=1, \ldots, n$. Let $f$ and $\tilde{r}$ be the density and the reverse hazard rate functions of $F$, respectively. If $x^{2} \widetilde{r}^{\prime}(x)$ is increasing, then

$$
\left(\lambda_{1}, \ldots, \lambda_{n}\right) \stackrel{m}{\geq}\left(\mu_{1}, \ldots, \mu_{n}\right) \Longrightarrow X_{n: n} \stackrel{r h}{\geq} Y_{n: n}
$$

It is proved in Khaledi et al. [97] that the conditions of Theorem 4.31 are satisfied by generalized gamma distribution with the parameters $p<1$ and $q>0$. That is, the reverse hazard rate of a parallel system consisting of independent components with $G G(p, q)$ lifetimes is Schur convex in the vector of scales parameters, when either $p<1$ and $q<1$ (i.e., $F$ is DFR,) or $p<1$ and $q>1$ (i.e., $F$ has an upside down bathtub failure rate).

Balakrishnan and Zhao [99] proved the following result for gamma distribution.

Theorem 4.32. Let $X_{1}, \ldots, X_{n}$ be independent gamma random variables with $X_{i}$ having shape parameter $0<r \leq 1$ and scale parameter $\lambda_{i}, i=1, \ldots, n$. Let $Y_{1}, \ldots, Y_{n}$ be a random sample of size $n$ from a gamma distribution with shape parameter $r$ and scale parameter $\lambda=\lambda \geq \tilde{\lambda}=\sqrt[n]{\prod_{i=1}^{n} \lambda_{i}}$, the geometric mean of $\lambda_{i}$ 's. Then

$$
X_{n: n} \geq_{h r} Y_{n: n}
$$

This result generalizes the results of Khaledi and Kochar [27] from exponential distribution to gamma distribution when the scale parameter is at most one.

\subsection{Dependence Orderings among Order Statistics from Independent Random Variables with Proportional Hazard Rates}

Sathe [100] proved that if $X_{1}, \ldots, X_{n}$ are independent exponential random variables with distinct parameters $\lambda_{1}, \lambda_{2}, \ldots, \lambda_{n}$, then for any $k=2, \ldots, n$, the Peasrson coefficient of correlation between $X_{k: n}$ and $X_{1: n}$ is maximum when the $\lambda_{i}$ 's are equal. The natural question is to see if we can extend this result to positive dependence orderings. Dolati et al. [58] studied 
this topic when the observations follow the proportional hazards rate model. They proved the following result for the extreme order statistics.

Theorem 4.33. Let $X_{1}, \ldots, X_{n}$ be independent continuous random variables following the PHR model. Let $Y_{1}, \ldots, Y_{n}$ be i.i.d. continuous random variables, then

$$
\left(X_{n: n} \mid X_{1: n}\right) \prec_{R T I}\left(Y_{n: n} \mid Y_{1: n}\right)
$$

They also wondered whether this result can be strengthened to more SI ordering. Genest et al. [60] gave a positive answer to this question by showing the following result.

Theorem 4.34. Let $X_{1}, \ldots, X_{n}$ be independent continuous random variables following the PHR model. Let $Y_{1}, \ldots, Y_{n}$ be i.i.d. continuous random variables, then

$$
\left(X_{n: n} \mid X_{1: n}\right) \prec_{S I}\left(Y_{n: n} \mid Y_{1: n}\right)
$$

It will be of interest to know whether Theorem 4.34 can be extended to other order statistics, that is, for $2 \leq j \leq n-1$,

$$
\left(X_{j: n} \mid X_{1: n}\right) \preceq_{\mathrm{SI}}\left(Y_{j: n} \mid Y_{1: n}\right)
$$

It is true for $j=2$. To prove our conjecture one needs to prove that

$$
X_{j: n}-X_{1: n} \leq_{\text {disp }} Y_{j: n}-Y_{1: n}
$$

whose proof is still elusive for $3 \leq j \leq n-1$.

It is also worth noting that Dolati et al. [58] got a nice bound for Kendall's tau of $\left(X_{n: n}, X_{1: n}\right)$ by using Theorem 4.33 ,

$$
\tau\left(X_{n: n}, X_{1: n}\right) \leq \frac{1}{2 n-1}
$$

\section{Stochastic Comparisons of Sample Spacings}

Spacings are of great interest in many areas of statistics, in particular, in the characterizations of distributions, goodness-of-fit tests, auction theory, life testing, and reliability models. A large number of goodness-of-fit tests are based on functions of sample spacings, see Balakrishnan and Rao [5, 6], Jammalamadaka and Taufer [101], and Jammalamadaka and Goria [102] for more references.

Let $X_{1}, \ldots, X_{n}$ be $n$ random variables. The random variables $D_{i: n}=X_{i: n}-X_{i-1: n}$ and $D_{i: n}^{*}=(n-i+1) D_{i: n}, i=1, \ldots, n$, with $X_{0: n} \equiv 0$, are respectively called spacings and normalized spacings. They are of great interest in various areas of statistics, in particular, in characterizations of distributions, goodness-of-fit tests, life testing, and reliability models. In the reliability context they correspond to times elapsed between successive failures of components in a system. In stochastic auction theory, $D_{n: n}$ and $D_{2: n}$ are of particular interest, which represent auction rents in buyer's auction and reverse auction in the second-price 
business auction (see [103]). In this section we consider the stochastic properties of spacings. In Section 5.1 we investigate stochastic order relations between successive spacings. In Section 5.2 we study the effect of changes in the scale parameters of the observations on stochastic properties of spacings when the observations are independent exponentials.

\subsection{Stochastic Orders between Successive Spacings in a Sample}

It is well known that the normalized spacings of a random sample from an exponential distribution are i.i.d. random variables having the same exponential distribution. Many authors have studied the stochastic properties of spacings from restricted families of distributions. Barlow and Proschan [104] proved the following result on stochastic ordering between the successive normalized spacings from DFR distributions.

Theorem 5.1. Let $X_{1}, \ldots, X_{n}$ be a random sample of size $n$ from a DFR distribution. Then

(a)

$$
D_{i: n}^{*} \leq_{s t} D_{i+1: n^{\prime}}^{*} \quad i=1, \ldots, n-1,
$$

(b)

$$
D_{i: n+1}^{*} \leq_{s t} D_{i: n^{\prime}}^{*} \quad n \geq i \text { for fixed } i .
$$

Similar results hold for the IFR case with the inequalities reversed in (a) and (b) above. Later Pledger and Proschan [15] partially extended this result to the case when the random variables are independent with proportional decreasing failure rates. Kim and David [70] have also obtained some results on spacings from IFR (DFR) distributions.

Kochar and Kirmani [19] strengthened this result from stochastic ordering to hazard rate ordering. They proved the following result.

Theorem 5.2. Let $X_{1}, \ldots, X_{n}$ be a random sample of size $n$ from a DFR distribution. Then

(a)

$$
D_{i: n}^{*} \leq_{h r} D_{i+1: n}^{*} \text { for } i=1, \ldots, n-1 \text {. }
$$

(b)

$$
D_{i: n+1}^{*} \leq_{h r} D_{i: n^{\prime}}^{*} \quad n \geq i \text { for fixed } i .
$$

Barlow and Proschan [104] have also shown that spacings of i.i.d. DFR random variables have also DFR distributions. Using this and the connection between hazard rate ordering and dispersive ordering, the proof of the next result follows from Theorem 2.12.

Theorem 5.3. If $X_{1}, \ldots, X_{n}$ is a random sample from a DFR distribution, then for $i=1, \ldots, n-1$,

(a) $D_{i: n}^{*} \leq_{\text {disp }} D_{i+1: n^{\prime}}^{*}$

(b) $D_{i: n+1}^{*} \leq_{\text {disp }} D_{i: n}^{*}$. 
The corresponding problem when the random variables are not identically distributed or not independent has also been studied by many researchers. Kochar and Kirmani [19] proved that when the joint density of the parent observations is convex, the joint density of the normalized spacings is ISO*. This will hold, in particular, when the $X_{i}^{\prime}$ s are independent (but not necessarily identical) with log-convex densities.

Theorem 5.4. Let the joint density $f_{\mathbf{X}}\left(x_{1}, \ldots, x_{n}\right)$ of $\mathbf{X}=\left(X_{1}, \ldots, X_{n}\right)$ be convex. Then the joint density of $\mathbf{D}=\left(D_{1: n}, \ldots, D_{n: n}\right)$ is ISO*

This result immediately leads to the following interesting result.

Theorem 5.5. Let $X_{1}, X_{2}, \ldots, X_{n}$ be independent random variables with log-convex densities. Then

$$
D_{1: n}^{*} \leq_{\ell r: j} D_{2: n}^{*} \leq_{\ell r: j} \cdots \leq_{\ell r: j} D_{n: n}^{*} .
$$

If a density is log-convex, it is DFR, but the converse is not true. Hence, Theorem 5.5 establishes a stronger ordering between the normalized spacings than does Theorem 5.2 under a stronger condition on the parent distributions. A related paper on this topic is by Nappo and Spizzichino [12].

One may wonder whether under the condition of Theorem 5.5 the result can be extended from joint likelihood ratio order to likelihood ratio order. The answer is negative as illustrated by a counterexample in Misra and van der Meulen [105].

Let $X_{1}, \ldots, X_{n}$ be independent exponential random variables with $X_{i}$ having failure rate $\lambda_{i}$ for $i=1, \ldots, n$. Hu and Xie [77] proved the following result.

Theorem 5.6. If $\lambda_{n+1} \leq[\geq] \lambda_{k}$ for $k=1, \ldots, n$, then

$$
\begin{gathered}
D_{n: n} \leq_{l r} D_{n+1: n+1}, \\
D_{1: n} \leq_{l r} D_{2: n+1}\left[D_{2: n+1} \leq_{l r} D_{2: n}\right] .
\end{gathered}
$$

If $\lambda_{i}+\lambda_{j} \geq \lambda_{k}$ for all distinct $i, j$ and $k$ then

$$
D_{n-1: n} \leq_{l r} D_{n: n,} \quad D_{n: n+1} \leq_{l r} D_{n: n} .
$$

They also showed that $D_{2: 3} \leq \operatorname{lr} D_{3: 3}$ for all $\lambda_{i}$ 's, and

$$
D_{1: n} \leq_{\operatorname{lr}} D_{2: n}, \quad n \geq 2
$$

Kochar and Korwar [20] conjectured that (5.3) can be extended to the case when $X_{1}, \ldots, X_{n}$ are independent exponential random variables with possibly different scale parameters. They proved their conjecture for $n=3$ and Torrado et al. [106] provided a proof of this conjecture for $n=4$, but the general case still remains an open problem. Torrado et al. [106] also proved that for any $n, D_{2: n}^{*} \leq h r D_{3: n}^{*}$. This topic has been extensively studied by Khaledi and Kochar [30], Wen et al. [107], Xu et al. [108], and Chen and Hu [109] in singleoutlier or multiple-outlier exponential models. 


\subsection{Comparing Spacings of Two Random Samples}

How does a variability ordering between two probability distributions affect the relative positioning of the observations in random samples from the two distributions? To answer this question, let $X_{1: n} \leq X_{2: n} \leq \cdots \leq X_{n: n}$ denote the order statistics of a random sample $X_{1}, X_{2}, \ldots, X_{n}$ from a distribution with distribution function $F$. Similarly, let $Y_{1: n} \leq Y_{2: n} \leq$ $\ldots \leq Y_{n: n}$ denote the order statistics of an independent random sample $Y_{1}, Y_{2}, \ldots, Y_{n}$ from a distribution with distribution function $G$. The corresponding spacings are defined by $D_{i: n} \equiv X_{i: n}-X_{i-1: n}$ and $H_{i: n} \equiv Y_{i: n}-Y_{i-1: n}$, for $\mathrm{i}=1,2, \ldots, n$, where $X_{0: n}=Y_{0: n} \equiv 0$. Bartoszewic [42] proved the following result.

Theorem 5.7. One has

$$
Y \leq_{\text {disp }} X \Longrightarrow\left(H_{1 n}, H_{2: n}, \ldots, H_{n: n}\right) \leq_{s t}\left(D_{1: n}, D_{2: n}, \ldots, D_{n: n}\right) .
$$

Some consequences of the this result as reported in Kochar [25] are as follows.

(a) For $1 \leq i<j \leq n$,

$$
X_{j: n}-X_{i: n} \geq_{s t} Y_{j: n}-Y_{i: n}
$$

In particular,

$$
X_{n: n}-X_{1: n} \geq_{s t} Y_{n: n}-Y_{1: n}
$$

(b) One has

$$
s_{X}^{2} \geq_{s t} s_{Y}^{2}
$$

where $s_{X}^{2}$ and $s_{Y}^{2}$ are the sample variances of the two samples.

(c) One has

$$
\eta_{X} \geq_{\text {st }} \eta_{Y}
$$

where

$$
\eta_{X}=\left[\left(\begin{array}{l}
n \\
2
\end{array}\right)\right]^{-1} \sum_{i<j}\left|X_{j: n}-X_{i: n}\right|
$$

is the Gini's mean difference for the $X$-sample. Similarly we define $\eta_{\gamma}$.

Kochar et al. [110] extended the implication (5.10) when dispersive ordering between $F$ and $G$ is replaced by either excess wealth order. They proved the following result. 
Theorem 5.8. If $Y \leq_{e w} X$, then, for all $1 \leq k \leq n-1$,

$$
X_{n: n}-X_{k: n} \geq_{i c x} Y_{n: n}-Y_{k: n} \Longrightarrow E\left[X_{n: n}-X_{k: n}\right] \leq E\left[Y_{n: n}-Y_{k: n}\right]
$$

Taking $k=1$ in this theorem gives us a result on the sample spacings,

$$
Y_{\mathrm{ew}} X \Longrightarrow X_{n: n}-X_{1: n} \geq_{\mathrm{icx}} Y_{n: n}-Y_{1: n} \text {. }
$$

It is well known that a random variable $X$ is NBUE (new better than used in expectation) if and only if $X \leq_{\mathrm{ew}} Y$, where $Y$ is exponential with the same mean as that of $X$ (see [111]). It follows directly from Theorem 5.8 that, if $X_{1}, \ldots, X_{n}$ is a random sample from a distribution which is NBUE, then,

$$
E\left[X_{n: n}-X_{k: n}\right] \leq\left[1+\cdots+\frac{1}{n-k}\right] E[X], \quad \text { for any } 1 \leq k \leq n-1 .
$$

\subsection{Comparing Spacings of Heterogeneous Exponential Samples}

Let $X_{1}, \ldots, X_{n}$ be independent exponential random variables with $X_{i}$ having hazard rate $\lambda_{i}$, $i=1, \ldots, n$, and let $Y_{1}, \ldots, Y_{n}$ be a random sample of size $n$ from an exponential distribution with common hazard rate $\lambda$. Kochar and Korwar [20] proved that the distribution function of $D_{k: n}^{*}$ for $i=2, \cdots, n$ is a mixture of independent exponential random variables with the density function

$$
f_{D_{k: n}^{*}}(x)=\sum_{\mathbf{r}} \frac{\prod_{i=1}^{n} \lambda_{i}}{\prod_{i=1}^{n} \sum_{j=i}^{n} \lambda_{r_{j}}}\left(\frac{\sum_{j=k}^{n} \lambda_{r_{j}}}{n-i+1}\right) \exp \left\{\frac{-x \sum_{j=k}^{n} \lambda_{r_{j}}}{n-i+1}\right\},
$$

where $\mathbf{r}$ extends over all of the permutations of $\{1,2, \ldots, n\}$. Hence, the distribution of $D_{k: n}^{*}$ could be represented as

$$
F_{D_{k: n}}(x)=\sum_{j \in \mathbf{r}} p_{j} F_{\lambda_{k j}^{*}}(x)
$$

where $j$ denotes a permutation of $\left(\lambda_{1}, \ldots, \lambda_{n}\right)$ belonging to $\mathbf{r}$ and

$$
\begin{aligned}
& p_{j}=\frac{\prod_{i=1}^{n} \lambda_{i}}{\prod_{i=1}^{n} \sum_{j=i}^{n} \lambda_{r_{j}}}, \\
& \lambda_{k j}^{*}=\frac{\sum_{j=k}^{n} \lambda_{r_{j}}}{(n-k+1)^{2}},
\end{aligned}
$$

and $F_{\lambda_{k j}^{*}}$ means an exponential distribution with hazard rate $\lambda_{k j}^{*}$.

Pledger and Proschan [15] considered the problem of stochastically comparing the spacings of nonidentical independent exponential random variables with those corresponding to stochastically comparable independent and identically distributed exponential random variables. 
Theorem 5.9 (see [15]). If $X_{1}, \ldots, X_{n}$ are independent exponential random variables with $X_{i}$ having hazard rate $\lambda_{i}, i=1, \ldots, n$, and $Y_{1}, \ldots, Y_{n}$ is a random sample of size $n$ from an exponential distribution with common hazard rate $\bar{\lambda}=\sum_{i=1}^{n} \lambda_{i} / n$, then

$$
H_{k: n}^{*} \leq_{s t} D_{k: n^{\prime}}^{*}
$$

where $H_{k: n}^{*}=(n-k+1)\left(Y_{k: n}-Y_{k-1: n}\right)$ for $k=1, \ldots, n$ are the normalized spacings of $Y_{i}^{\prime}$ s, and $Y_{0: n} \equiv 0$.

Kochar and Korwar [20] strengthened and extended this result from stochastic ordering to likelihood ratio ordering and dispersive ordering.

Theorem 5.10. Under the conditions of Theorem 5.9,

$$
H_{k: n}^{*} \leq_{l r} D_{k: n^{\prime}}^{*} \quad H_{k: n}^{*} \leq_{\text {disp }} D_{k: n^{\prime}}^{*} \quad k=1, \ldots, n .
$$

Kochar and Rojo [21] further strengthened Theorem 5.10 to multivariate likelihood ratio order.

Theorem 5.11. Under the conditions of Theorem 5.9,

$$
\left(H_{1: n^{\prime}}^{*}, \ldots, H_{n: n}^{*}\right) \leq_{l r}\left(D_{1: n^{\prime}}^{*}, \ldots, D_{n: n}^{*}\right) .
$$

As a consequence, it follows that

$$
Y_{j: n}-Y_{i: n} \leq_{\mathrm{st}} X_{j: n}-X_{i: n} \quad \forall 1 \leq i<j \leq n
$$

Kochar and $\mathrm{Xu}$ [112] established the following results providing sufficient and necessary conditions for stochastically comparing $D_{k: n}$ and $H_{k: n}$ according to likelihood ratio and reverse hazard rate orderings.

Theorem 5.12. Let $X_{1}, \ldots, X_{n}$ be independent exponential random variables with $X_{i}$ having hazard rate $\lambda_{i}, i=1, \ldots, n$, and $Y_{1}, \ldots, Y_{n}$ be a random sample of size $n$ from an exponential distribution with common hazard rate $\lambda$. Then, for $k \geq 2$,

(a) $H_{k: n} \leq_{l r} D_{k: n}$ or

(b) $H_{k: n} \leq_{r h} D_{k: n}$;

if and only if

$$
\lambda \geq \frac{1}{n-k+1} \frac{\sum_{\mathbf{r}}\left(\left(\prod_{i=1}^{n} \lambda_{i}\right) /\left(\prod_{i=1}^{n} \sum_{j=i}^{n} \lambda_{r_{j}}\right)\right)\left(\sum_{j=k}^{n} \lambda_{r_{j}}\right)^{2}}{\sum_{\mathbf{r}}\left(\left(\prod_{i=1}^{n} \lambda_{i}\right) /\left(\prod_{i=1}^{n} \sum_{j=i}^{n} \lambda_{r_{j}}\right)\right) \sum_{j=k}^{n} \lambda_{r_{j}}} .
$$

Analogously, we have an equivalent necessary and sufficient condition for hazard rate order, dispersive order, and stochastic order between $D_{k: n}$ and $H_{k: n}$ as proved in Kochar and $\mathrm{Xu}[112]$. 
Theorem 5.13. Let $X_{1}, \ldots, X_{n}$ be independent exponential random variables with $X_{i}$ having hazard rate $\lambda_{i}, i=1, \ldots, n$, and $Y_{1}, \ldots, Y_{n}$ be a random sample of size $n$ from an exponential distribution with common hazard rate $\lambda$. Then, for $k \geq 2$,

(a) $H_{k: n} \leq{ }_{h r} D_{k: n}$,

(b) $H_{k: n} \leq$ disp $D_{k: n}$ or

(c) $H_{k: n} \leq_{s t} D_{k: n}$

if and only if

$$
\lambda \geq \frac{1}{n-k+1} \sum_{\mathbf{r}} \frac{\prod_{i=1}^{n} \lambda_{i}}{\prod_{i=1}^{n} \sum_{j=i}^{n} \lambda_{r_{j}}} \sum_{j=k}^{n} \lambda_{r_{j}} .
$$

The next result is also from Kochar and $\mathrm{Xu}$ [112].

Theorem 5.14. Let $X_{1}, \ldots, X_{n}$ be independent exponential random variables with $X_{i}$ having hazard rate $\lambda_{i}, i=1, \ldots, n$, and $Y_{1}, \ldots, Y_{n}$ be a random sample of size $n$ from an exponential distribution with common hazard rate $\lambda$. Then, for $k \geq 2$,

(a) $H_{k: n} \leq_{m r l} D_{k: n}$,

(b) $H_{k: n} \leq_{R S} D_{k: n}$ or

(c) $E H_{k: n} \leq E D_{k: n}$

if and only if

$$
\lambda \geq \frac{1}{n-k+1} \frac{1}{\sum_{\mathbf{r}}\left(\left(\prod_{i=1}^{n} \lambda_{i}\right) /\left(\sum_{j=k}^{n} \lambda_{r_{j}} \prod_{i=1}^{n} \sum_{j=i}^{n} \lambda_{r_{j}}\right)\right)} .
$$

Example 5.15. Suppose $X_{1}, X_{2}, X_{3}$ are independent exponential random variables with parameters $\lambda_{1}, \lambda_{2}, \lambda_{3}$, respectively; and $Y_{1}, Y_{2}, Y_{3}$ are i.i.d. exponential random variables with parameter $\lambda$. Then, from Theorem 5.13,

$$
Y_{3: 3}-Y_{2: 3} \leq_{\mathrm{hr}} X_{3: 3}-X_{2: 3}
$$

if and only if

$$
\lambda \geq \lambda_{\mathrm{hr}}=\frac{2 \lambda_{1} \lambda_{2} \lambda_{3}}{\lambda_{1}+\lambda_{2}+\lambda_{3}}\left(\frac{1}{\lambda_{2}+\lambda_{3}}+\frac{1}{\lambda_{1}+\lambda_{3}}+\frac{1}{\lambda_{1}+\lambda_{2}}\right) .
$$

From Theorem 5.12,

$$
Y_{3: 3}-Y_{2: 3} \leq_{\operatorname{lr}} X_{3: 3}-X_{2: 3}
$$

if and only if

$$
\lambda \geq \lambda_{\mathrm{lr}}=\frac{3}{2}\left(\frac{1}{\lambda_{2}+\lambda_{3}}+\frac{1}{\lambda_{1}+\lambda_{3}}+\frac{1}{\lambda_{1}+\lambda_{2}}\right)^{-1} .
$$


From Theorem 5.14

$$
Y_{3: 3}-Y_{2: 3} \leq_{\mathrm{RS}} X_{3: 3}-X_{2: 3}
$$

if and only if

$$
\lambda \geq \lambda_{\mathrm{RS}}=\left[\frac{\lambda_{1} \lambda_{2} \lambda_{3}}{\lambda_{1}+\lambda_{2}+\lambda_{3}}\left(\frac{\lambda_{2}^{2}+\lambda_{3}^{2}}{\left(\lambda_{2}+\lambda_{3}\right) \lambda_{2}^{2} \lambda_{3}^{2}}+\frac{\lambda_{1}^{2}+\lambda_{3}^{2}}{\left(\lambda_{1}+\lambda_{3}\right) \lambda_{1}^{2} \lambda_{3}^{2}}+\frac{\lambda_{2}^{2}+\lambda_{1}^{2}}{\left(\lambda_{2}+\lambda_{1}\right) \lambda_{2}^{2} \lambda_{1}^{2}}\right)\right]^{-1} .
$$

It is worth noting that from (2.7), it follows that

$$
\lambda_{l r} \geq \lambda_{\mathrm{hr}} \geq \lambda_{\mathrm{RS}}
$$

For example, let

$$
\lambda_{1}=1, \quad \lambda_{2}=2, \quad \lambda_{3}=3 .
$$

Then,

$$
\lambda_{\mathrm{lr}}=1.91489>\lambda_{\mathrm{hr}}=1.56667>\lambda_{\mathrm{RS}}=1.30435 .
$$

Recently Balakrishnan and $\mathrm{Xu}$ [113] have established the following interesting result which compares the degree of skewness between sample spacings according to star ordering.

Theorem 5.16. Let $X_{1}, \ldots, X_{n}$ be independent exponential random variables with $X_{i}$ having hazard rate $\lambda_{i}, i=1, \ldots, n$, and $Y_{1}, \ldots, Y_{n}$ be a random sample of size $n$ from an exponential distribution with common hazard rate $\lambda$. Then, for $k \geq 2$,

$$
H_{k: n} \leq_{*} D_{k: n} .
$$

Torrado and Lillo [114] considered the problem of stochastically comparing the spacings of two heterogenous samples with independent exponential random variables. They obtained sufficient conditions on the parameters for such comparisons to hold.

\subsection{Stochastic Comparisons of Sample Ranges of Two Heterogeneous Samples}

Sample range is one of the simple criteria for comparing variabilities among distributions and hence it is important to study its stochastic properties. First we study the stochastic properties of the range of a random sample from a continuous distribution. Let $X_{1}, \ldots, X_{n}$ be a random sample from $F$ and let $Y_{1}, \ldots, Y_{n}$ be an independent random sample from another distribution G. It follows from Lemma 3(c) of Bartoszewic [42] that $X \geq_{\text {disp }} Y \Rightarrow X_{n: n}-X_{1: n} \geq_{\text {st }} Y_{n: n}-Y_{1: n}$. This observation along with Theorem 2.12 (a) leads to the following theorem. 
Theorem 5.17. Let $X \geq_{h r} Y$ and let either F or $G$ be DFR. Then

$$
Y_{n: n}-Y_{1: n} \leq_{s t} X_{n: n}-X_{1: n}
$$

Kochar and Rojo [21] proved the following result on stochastic properties of the sample range when the observations are independent exponentials with unequal parameters.

Theorem 5.18. Let $X_{1}, \ldots, X_{n}$ be independent exponential random variables with $X_{i}$ having hazard rate $\lambda_{i}, i=1, \ldots, n$, and let $Y_{1}, \ldots, Y_{n}$ be a random sample of size $n$ from an exponential distribution with common hazard rate $\bar{\lambda}=\sum_{i=1}^{n} \lambda_{i} / n$, then,

$$
Y_{n: n}-Y_{1: n} \leq_{s t} X_{n: n}-X_{1: n}
$$

The next theorem due to Khaledi and Kochar [28] improves upon this result.

Theorem 5.19. Let $X_{1}, \ldots, X_{n}$ be independent exponential random variables with $X_{i}$ having hazard rate $\lambda_{i}, i=1, \ldots, n$, and let $Y_{1}, \ldots, Y_{n}$ be a random sample of size $n$ from an exponential distribution with common hazard rate $\tilde{\lambda}=\left(\prod_{i=1}^{n} \lambda_{i}\right)^{1 / n}$, the geometric mean of the $\lambda^{\prime} s$. Then,

$$
Y_{n: n}-Y_{1: n} \leq_{s t} X_{n: n}-X_{1: n}
$$

As a consequence of this result we get the following upper bound on the distribution function of the sample range in terms $\tilde{\lambda}$, the geometric mean of the parameters.

Corollary 5.20. Let $X_{1}, \ldots, X_{n}$ be independent exponential random variables with $X_{i}$ having hazard rate $\lambda_{i}, i=1, \ldots, n$. Then for $x>0$,

$$
P\left[X_{n: n}-X_{1: n} \leq x\right] \leq\left[1-e^{-\tilde{\lambda} x}\right]^{n-1}
$$

This bound is better than the one obtained in Kochar and Rojo [21] in terms of $\bar{\lambda}$, since the expression on the R.H.S. of (5.41) is increasing in $\tilde{\lambda}$ and $\tilde{\lambda} \leq \bar{\lambda}$.

Recently, Zhao and Li [90] obtained a necessary and sufficient condition for stochastically comparing sample ranges from heterogeneous and homogeneous exponential samples.

Theorem 5.21. Let $X_{1}, \ldots, X_{n}$ be independent exponential random variables with $X_{i}$ having hazard rate $\lambda_{i}, i=1, \ldots, n$, and let $Y_{1}, \ldots, Y_{n}$ be a random sample of size $n$ from an exponential distribution with common hazard rate $\lambda$, then,

$$
Y_{n: n}-Y_{1: n} \leq_{s t} X_{n: n}-X_{1: n} \Longleftrightarrow \lambda \geq \hat{\jmath}=\left(\frac{\prod_{i=1}^{n} \lambda_{i}}{\bar{\jmath}}\right)^{1 /(n-1)}
$$

where $\bar{\lambda}=\sum_{i=1}^{n} \lambda_{i} / n$.

Note that $\hat{\lambda} \leq \tilde{\lambda}$, which improves upon the results of Theorem 5.19. 
Kochar and $\mathrm{Xu}$ [85] partially strengthened Theorem 5.18 from stochastic order to reverse hazard rate order.

Theorem 5.22. Let $X_{1}, \ldots, X_{n}$ be independent exponential random variables with $X_{i}$ having hazard rate $\lambda_{i}, i=1, \ldots, n$. Let $Y_{1}, \ldots, Y_{n}$ be a random sample of size $n$ from an exponential distribution with common hazard rate $\bar{\lambda}=\sum_{i=1}^{n} \lambda_{i} / n$. Then

$$
Y_{n: n}-Y_{1: n} \leq_{r h} X_{n: n}-X_{1: n}
$$

They also proved the following theorem for the PHR model.

Theorem 5.23. Let $X_{1}, \ldots, X_{n}$ be independent random variables with $X_{i}$ having survival function $\bar{F}^{\lambda_{i}}, i=1, \ldots, n$. Let $Y_{1}, \ldots, Y_{n}$ be a random sample with common population survival distribution $\bar{F}^{\bar{\lambda}}$, where $\bar{\lambda}=\sum_{i=1}^{n} \lambda_{i} / n$, then

$$
Y_{n: n}-Y_{1: n} \leq_{s t} X_{n: n}-X_{1: n}
$$

The following result of Genest et al. [60] establishes likelihood ration ordering between the sample ranges and improves upon all the previously known results on this problem.

Theorem 5.24. Let $X_{1}, \ldots, X_{n}$ be independent exponential random variables with $X_{i}$ having hazard rate $\lambda_{i}, i=1, \ldots, n$, and $Y_{1}, \ldots, Y_{n}$ be a random sample of size $n$ from an exponential distribution with common hazard rate $\bar{\lambda}=\sum_{i=1}^{n} \lambda_{i} / n$. Let $\widetilde{r}_{X}(x)$ and $\widetilde{r}_{Y}(x)$ be reversed hazard rates of $X_{n: n}-X_{1: n}$ and $Y_{n: n}-Y_{1: n}$, respectively. Then

$$
\frac{\tilde{r}_{X}(x)}{\widetilde{r}_{Y}(x)} \text { is increasing. }
$$

The following results follow as consequences as shown in that paper.

Corollary 5.25. Let $X_{1}, \ldots, X_{n}$ be independent exponential random variables with $X_{i}$ having hazard rate $\lambda_{i}, i=1, \ldots, n$. Let $Y_{1}, \ldots, Y_{n}$ be a random sample of size $n$ from an exponential distribution with common hazard rate $\bar{\lambda}$, then,

(a)

$$
Y_{n: n}-Y_{1: n} \leq_{l r} X_{n: n}-X_{1: n}
$$

(b)

$$
Y_{n: n}-Y_{1: n} \leq_{d i s p} X_{n: n}-X_{1: n}
$$

Balakrishnan and $\mathrm{Xu}$ [113] established a star ordering result between sample ranges as reported in the next theorem. 
Theorem 5.26. Let $X_{1}, \ldots, X_{n}$ be independent exponential random variables with $X_{i}$ having hazard rate $\lambda_{i}, i=1, \ldots, n$ and let $Y_{1}, \ldots, Y_{n}$ be a random sample of size $n$ from an exponential distribution with common hazard rate $\lambda$. Then

$$
Y_{n: n}-Y_{1: n} \leq_{*} X_{n: n}-X_{1: n}
$$

Let $X_{1}, \ldots, X_{n}$ be independent exponential random variables with $X_{i}$ having hazard rate $\lambda_{i}, i=1, \ldots, n$ and let $Y_{1}, \ldots, Y_{n}$ be an another set of independent exponential random variables with hazard rates $\mu_{1}, \ldots, \mu_{n}$, respectively. Kochar and Rojo [20] proved that

$$
\left(\lambda_{1}, \ldots, \lambda_{n}\right) \stackrel{m}{\geq}\left(\mu_{1}, \ldots, \mu_{n}\right) \Longrightarrow X_{n: n}-X_{1: n} \geq_{s t} Y_{n: n}-Y_{1: n}
$$

Recently Ding et al. [115] strengthened (5.49) from stochastic ordering to reverse hazard rate ordering. Then also proved that under the above set up,

$$
\left(\log \lambda_{1}, \ldots, \log \lambda_{n}\right) \stackrel{m}{\geq}\left(\mu_{1}, \ldots, \mu_{n}\right) \Longrightarrow X_{n: n}-X_{1: n} \geq_{s t} Y_{n: n}-Y_{1: n}
$$

They also extended (5.49) to the PHR model.

\subsection{Dependence Orderings among Spacings}

It is known that spacings of a random sample from a DFR (decreasing failure rate) distribution are conditionally increasing in sequence (cf., [49, page 151]). Karlin and Rinott [54] have pointed out that if the DFR assumption is strengthened to assume that the parent distribution has a log-convex density, then the spacings have the corresponding stronger property of being $\mathrm{MTP}_{2}$ dependent. Khaledi and Kochar [116] extended this result to the case when the random variables $X_{1}, \ldots, X_{n}$ are dependent. It is proved that if the joint pdf of $X_{i}{ }^{\prime} \mathrm{S}$ is permutation symmetric, $\mathrm{TP}_{2}$ in pairs and log-convex in each argument, then their spacings are $\mathrm{MTP}_{2}$ dependent. They also studied the dependence properties of spacings of independent but non-identically distributed exponential random variables and showed with the help of a counterexample that in this case the spacings may not be $\mathrm{MTP}_{2}$ dependent. Khaledi and Kochar [116] proved that in the case of a single-outlier when all except one of the parameters are equal, the spacings are $\mathrm{MTP}_{2}$ dependent. A consequence of this result is that in this case $\operatorname{var}\left(X_{1: n}\right) \leq \operatorname{var}\left(X_{2: n}\right) \leq \cdots \leq \operatorname{var}\left(X_{n: n}\right)$. They also proved that in the case of multiple-outliers model any pair of consecutive spacings, $D_{i: n}$ and $D_{i+1: n}$ are $\mathrm{TP}_{2}$ dependent for $i=1, \ldots, n-1$.

\section{Applications}

\subsection{Type-II Censoring}

If $n$ items are put on life test and the test terminated at the time of $r$ th failure, then

$$
T_{r n}=\sum_{i=1}^{r}(n-i+1)\left(X_{i: n}-X_{i-1: n}\right)=\sum_{i=1}^{r} D_{i: n}^{*}
$$


represents the total time on test (TTT), which is a well-known concept in statistical reliability. As pointed out in Epstein [117], if the component lifetimes are i.i.d. exponential with mean $\theta$ or equivalently the failure rate $\lambda=1 / \theta$, the best estimator (minimum variance unbiased estimator) of $\theta$ is

$$
\widehat{\theta}=\frac{T_{r n}}{r}
$$

It is well-known that (cf., [117]),

$$
\frac{2 T_{r n}}{r} \stackrel{L}{\longrightarrow} X_{2 r}^{2}
$$

where $\chi_{2 r}^{2}$ denotes a chi-square distribution with $2 r$ degrees of freedom. Based on this result, many estimation and testing statistical procedures have been established. However, if the components are actually from different exponential distributions with failure rates $\lambda_{1}^{*}, \ldots, \lambda_{n^{\prime}}^{*}$ then it follows from Theorem 5.11 and Theorem 6.E.8 of Shaked and Shanthikumar [2] that

$$
\widehat{\theta}^{*} \geq_{\mathrm{st}} \widehat{\theta},
$$

where

$$
\widehat{\theta}^{*}=\frac{\sum_{i=1}^{r} D_{i: n}^{*}}{r}
$$

and $\lambda=\sum_{i=1}^{n} \lambda_{i}^{*} / n$. This means that based on TTT statistic, one would overestimate the average lifetime of components using heterogeneous exponential components.

\subsection{Reliability}

An $n$-component system that fails if and only if at least $k$ of the $n$ components fail is called a $k$-out-of- $n$ : F system. The lifetime of such a system could be represented as $X_{k: n}$. The $k$-out-of$n$ system structure is a very popular type of redundancy in fault tolerant systems with wide applications in industrial and military systems. For two different systems say a $k$-out-of- $n$ : F system and a $k+1$-out-of- $n$ : F system, the engineer may be interested in the additional lifetime $X_{k+1: n}-X_{k: n}$ for the system design and the cost purpose. Due to the complicated distribution form, one may provide a sharp bound on the survival function of this based on Theorem 5.13.

For example, a plane has four engines, and a minimum of three engines are required for the plane to work. Hence, this plane is a 2-out-of-4: F system. If the engineer wants to improve the system to the 3-out-of-4: F system, he/she has to consider the cost and the reliability of improvement simultaneously. Hence, it is important to estimate the survival probability and the mean additional lifetime $X_{3: 4}-X_{2: 4}$. Theorem 5.13 provides a lower bound for the survival function of the additional lifetime. Let $\left(\lambda_{1}, \lambda_{2}, \lambda_{3}, \lambda_{4}\right)=(1,2,2.5,3)$, then,

$$
P\left(X_{3: 4}-X_{2: 4}>x\right) \geq e^{-0.955 x}
$$

Using Theorem 5.14, the following sharp lower bound could be established,

$$
E\left(X_{3: 4}-X_{2: 4}\right) \geq 1.0879
$$


Table 1

\begin{tabular}{lccc}
\hline$\lambda$ & $\mathrm{lr} / \mathrm{rh}$ & $\mathrm{hr} / \mathrm{disp} / \mathrm{st}$ & $\mathrm{mrl} / \mathrm{RS} / E$ \\
\hline$D_{2: 4}$ & 2.060897 & 2.039216 & 2.019271 \\
$D_{3: 4}$ & 1.988454 & 1.909626 & 1.838388 \\
$D_{4: 4}$ & 2.009722 & 1.674501 & 1.382051 \\
\hline
\end{tabular}

In Table 1, we list the lower bounds on $\lambda^{\prime}$ s for various partial orders to hold in Theorems 5.12-5.14.

The values above are comparable to the arithmetic mean 2.125, geometric mean 1.96799, and harmonic mean 1.791045 of $\lambda_{i}$ 's. It is interesting to note that all of the values are less than the arithmetic mean, which coincides with the condition in Theorem 5.11.

\section{References}

[1] A. Muller and D. Stoyan, Comparison Methods for Stochastic Models and Risks, John Wiley \& Sons, New York, NY, USA, 2002.

[2] M. Shaked and J. G. Shanthikumar, Stochastic Orders, Springer Series in Statistics, Springer, New York, NY, USA, 2007.

[3] H. A. David and H. N. Nagaraja, Order Statistics, John Wiley \& Sons, New York, NY, USA, 3rd edition, 2003.

[4] B. C. Arnold, N. Balakrishnan, and H. N. Nagaraja, A First Course in Order Statistics, John Wiley \& Sons, New York, NY, USA, 1992.

[5] N. Balakrishnan and C. R. Rao, Handbook of Statistics 16: Order Statistics: Theory and Methods, Elsevier, New York, NY, USA, 1998.

[6] N. Balakrishnan and C. R. Rao, Handbook of Statistics 17: Order Statistics: Applications, Elsevier, New York, NY, USA, 1998.

[7] P. K. Sen, "A note on order statistics for heterogeneous distributions," The Annals of Mathematical Statistics, vol. 41, no. 6, pp. 2137-2139, 1970.

[8] M. Shaked and Y. L. Tong, "Stochastic ordering of spacings from dependent random variables," Inequalities in Statistics and Probability, vol. 5, pp. 141-149, 1984.

[9] R. B. Bapat and M. I. Beg, "Order statistics for nonidentically distributed variables and permanents," Sankhyā B, vol. 51, no. 1, pp. 79-93, 1989.

[10] P. J. Boland, M. Hollander, K. Joag-Dev, and S. C. Kochar, "Bivariate dependence properties of order statistics," Journal of Multivariate Analysis, vol. 56, no. 1, pp. 75-89, 1996.

[11] S. C. Kochar, "Dispersive ordering of order statistics," Statistics and Probability Letters, vol. 27, no. 3, pp. 271-274, 1996.

[12] G. Nappo and F. Spizzichino, "Ordering properties of the TTT-plot of lifetimes with schur joint densities," Statistics and Probability Letters, vol. 39, no. 3, pp. 195-203, 1998.

[13] P. J. Boland, M. Shaked, and J. G. Shanthikumar, "Stochastic ordering of order statistics," in Handbook of Statistics 16 - Order Statistics : Theory and Methods, N. Balakrishnan and C. R. Rao, Eds., pp. 89-103, Elsevier, New York, NY, USA, 1998.

[14] N. Balakrishnan, "Permanents, order statistics, outliers, and robustness," Revista Matemática Complutense, vol. 20, no. 1, pp. 7-107, 2007.

[15] P. Pledger and F. Proschan, "Comparisons of order statistics and of spacings from heterogeneous distributions," in Optimizing Methods in Statistics, J. S. Rustagi, Ed., pp. 89-113, Academic Press, New York, NY, USA, 1971.

[16] F. Proschan and J. Sethuraman, "Stochastic comparisons of order statistics from heterogeneous populations, with applications in reliability," Journal of Multivariate Analysis, vol. 6, no. 4, pp. 608616, 1976.

[17] R. B. Bapat and S. C. Kochar, "On likelihood-ratio ordering of order statistics," Linear Algebra and Its Applications, vol. 199, no. 1, pp. 281-291, 1994.

[18] P. J. Boland, E. El-Neweihi, and F. Proschan, "Applications of the hazard rate ordering in reliability and order statistics," Journal of Applied Probability, vol. 31, no. 1, pp. 180-192, 1994. 
[19] S. C. Kochar and S. N. U. A. Kirmani, "Some results on normalized spacings from restricted families of distributions," Journal of Statistical Planning and Inference, vol. 46, no. 1, pp. 47-57, 1995.

[20] S. C. Kochar and R. Korwar, "Stochastic orders for spacings of heterogeneous exponential random variables," Journal of Multivariate Analysis, vol. 57, no. 1, pp. 69-83, 1996.

[21] S. C. Kochar and J. Rojo, "Some new results on stochastic comparisons of spacings from heterogeneous exponential distributions," Journal of Multivariate Analysis, vol. 59, no. 2, pp. 272-281, 1996.

[22] R. Dykstra, S. C. Kochar, and J. Rojo, "Stochastic comparisons of parallel systems of heterogeneous exponential components," Journal of Statistical Planning and Inference, vol. 65, no. 2, pp. 203-211, 1997.

[23] S. C. Kochar and C. Ma, "Dispersive ordering of convolutions of exponential random variables," Statistics and Probability Letters, vol. 43, no. 3, pp. 321-324, 1999.

[24] J. L. Bon and E. Păltănea, "Ordering properties of convolutions of exponential random variables," Lifetime Data Analysis, vol. 5, no. 2, pp. 185-192, 1999.

[25] S. C. Kochar, "On stochastic orderings between distributions and their sample spacings," Statistics and Probability Letters, vol. 42, no. 4, pp. 345-352, 1999.

[26] B.-E. Khaledi and S. C. Kochar, "Stochastic orderings between distributions and their sample spacings-II," Statistics and Probability Letters, vol. 44, no. 2, pp. 161-166, 1999.

[27] B.-E. Khaledi and S. C. Kochar, "On dispersive ordering between order statistics in one-sample and two-sample problems," Statistics and Probability Letters, vol. 46, no. 3, pp. 257-261, 2000.

[28] B.-E. Khaledi and S. C. Kochar, "Some new results on stochastic comparisons of parallel systems," Journal of Applied Probability, vol. 37, no. 4, pp. 1123-1128, 2000.

[29] B. Khaledi and S. C. Kochar, "Sample range-some stochastic comparisons results," Calcutta Statistical Association Bulletin, vol. 50, pp. 283-291, 2000.

[30] B.-E. Khaledi and S. C. Kochar, "Stochastic properties of spacings in a single-outlier exponential model," Probability in the Engineering and Informational Sciences, vol. 15, no. 3, pp. 401-408, 2001.

[31] S. M. Ross, Stochastic Processes, John Wiley \& Sons, New York, NY, USA, 1983.

[32] J. G. Shanthikumar and D. D. Yao, "Bivariate characterization of some stochastic order relations," Advances in Applied Probability, vol. 23, pp. 642-659, 1991.

[33] M. Hollander, F. Proschan, and J. Sethuraman, "Functions decreasing in transposition and their applications in ranking problems," The Annals of Statistics, vol. 5, no. 4, pp. 722-733, 1977.

[34] A. W. Marshall, I. Olkin, and B. Arnold, Inequalities : Theory of Majorization and Its Applications, Springe, New York, NY, USA, 2nd edition, 2010.

[35] T. Robertson and F. T. Wright, "On measuring the conformity of a parameter set to a trend, with applications," Annals of Statistics, vol. 10, no. 4, pp. 1234-1245, 1982.

[36] K. Doksum, "Star shaped transformations and the power of rank tests," Annals of Mathematical Statistics, vol. 40, no. 4, pp. 1167-1176, 1969.

[37] J. V. Deshpande and S. C. Kochar, "Dispersive ordering is the same as tail ordering," Advances in Appllied Probability, vol. 15, pp. 686-687, 1983.

[38] D. J. Saunders, "Dispersive ordering of distributions," Advances in Applied Probability, vol. 16, pp. 693-694, 1984.

[39] I. W. Saunders and P. A. P. Moran, “On quantiles of the gamma and F distributions," Journal of Applied Probability, vol. 15, no. 2, pp. 426-432, 1978.

[40] T. Lewis and J. W. Thompson, "Dispersive distribution and the connection between dispersivity and strong unimodality," Journal of Applied Probability, vol. 18, no. 1, pp. 76-90, 1981.

[41] I. Bagai and S. C. Kochar, "On tail-ordering and comparison of failure rates," Communications in Statistics, vol. 15, no. 4, pp. 1377-1388, 1986.

[42] J. Bartoszewicz, "Dispersive ordering and the total time on test transformation," Statistics and Probability Letters, vol. 4, no. 6, pp. 285-288, 1986.

[43] J. Bartoszewicz, "A note on dispersive ordering defined by hazard functions," Statistics and Probability Letters, vol. 6, no. 1, pp. 13-16, 1987.

[44] S. C. Kochar, "Distribution-free comparison of two probability distributions with reference to their hazard rates," Biometrika, vol. 66, no. 3, pp. 437-441, 1979.

[45] J. Jeon, S. C. Kochar, and C. G. Park, "Dispersive ordering-some applications and examples," Statistical Papers, vol. 47, no. 2, pp. 227-247, 2006.

[46] J. M. Fernandez-Ponce, S. C. Kochar, and J. Muñoz-Perez, "Partial orderings of distributions based on right-spread functions," Journal of Applied Probability, vol. 35, no. 1, pp. 221-228, 1998.

[47] M. Shaked and J. G. Shanthikumar, "Two variability orders," Probability in the Engineering and Informational Sciences, vol. 12, no. 1, pp. 1-23, 1998. 
[48] W. R. van Zwet, Convex Transformations of Random Variables, vol. 7 of Mathematical Centre Tracts, Mathematical Centre, Amsterdam, The Netherlands, 2nd edition, 1964.

[49] R. E. Barlow and F. Proschan, Statistical Theory of Reliability and Life Testing, Silver Spring, Maryland, MD, USA, 1981.

[50] S. C. Kochar, "On extensions of DMRL and related partial orderings of life distributions," Stochastic Models, vol. 5, no. 2, pp. 235-246, 1989.

[51] E. L. Lehmann, "Some concepts of dependence," Annals of Mathematical Statistics, vol. 37, no. 5, pp. 1137-1153, 1966.

[52] J. H. B. Kemperman, "On the FKG-inequality for measures on a partially ordered space," Indagationes Mathematicae, vol. 39, no. 4, pp. 313-331, 1977.

[53] H. W. Block and M. L. Ting, "Some concepts of multivariate dependence," Communications in Statistics, vol. 10, no. 8, pp. 749-762, 1981.

[54] S. Karlin and Y. Rinott, "Classes of orderings of measures and related correlation inequalities. I. multivariate totally positive distributions," Journal of Multivariate Analysis, vol. 10, no. 4, pp. 467$498,1980$.

[55] H. Joe, Multivariate Models and Dependence Concepts, Chapman \& Hall, London, UK, 1997.

[56] R. B. Nelsen, "On measures of association as measures of positive dependence," Statistics and Probability Letters, vol. 14, no. 4, pp. 269-274, 1992.

[57] J. Avérous, C. Genest, and S. C. Kochar, "On the dependence structure of order statistics," Journal of Multivariate Analysis, vol. 94, no. 1, pp. 159-171, 2005.

[58] A. Dolati, C. Genest, and S. C. Kochar, "On the dependence between the extreme order statistics in the proportional hazards model," Journal of Multivariate Analysis, vol. 99, no. 5, pp. 777-786, 2008.

[59] R. B. Nelsen, An Introduction to Copulas, Lecture Notes in Statistics, Springer, New York, NY, USA, 2nd edition, 2006.

[60] C. Genest, S. C. Kochar, and M. Xu, "On the range of heterogeneous samples," Journal of Multivariate Analysis, vol. 100, no. 8, pp. 1587-1592, 2009.

[61] M. Z. Raqab and W. A. Amin, "Some ordering results on order statistics and record values," IAPQR Transactions, vol. 21, pp. 1-8, 1996.

[62] H. A. David and R. A. Groeneveld, "Measures of local variation in a distribution: expected length of spacings and variances of order statistics," Biometrika, vol. 69, no. 1, pp. 227-232, 1982.

[63] B. C. Arnold and J. A. Villasenor, "Lorenz ordering of order statistics," in Stochastic Orders and Decision under Risk, vol. 19 of IMS Lecture Notes Monograph Series, pp. 38-47, Institute of Mathematical Statistics, Hayward, Calif, USA, 1991.

[64] B. C. Arnold and H. N. Nagaraja, "Lorenz ordering of exponential order statistics," Statistics and Probability Letters, vol. 11, no. 6, pp. 485-490, 1991.

[65] B. Wilfling, "Lorenz ordering of power-function order statistics," Statistics and Probability Letters, vol. 30, no. 4, pp. 313-319, 1996.

[66] C. Kleiber, "Variability ordering of heavy-tailed distributions with applications to order statistics," Statistics and Probability Letters, vol. 58, no. 4, pp. 381-388, 2002.

[67] S. C. Kochar, "Lorenz ordering of order statistics," Statistics and Probability Letters, vol. 76, no. 17, pp. 1855-1860, 2006.

[68] P. J. Bickel, "Some contributions to the theory of order statistics," in Fifth Berkeley Symposium on Mathematics and Statistics, L. M. LeCam and J. Neyman, Eds., vol. 1, pp. 575-591, University of California Press, Berkeley, Calif, USA, 1967.

[69] J. W. Tukey, "A problem of berkson, and minimum variance orderly estimators," The Annals of Mathematical Statististis, vol. 29, no. 2, pp. 588-592, 1958.

[70] S. H. Kim and H. A. David, "On the dependence structure of order statistics and concomitants of order statistics," Journal of Statistical Planning and Inference, vol. 24, no. 3, pp. 363-368, 1990.

[71] M. Scarsini, “On measures of concordance," Stochastica, vol. 8, no. 3, pp. 201-218, 1984.

[72] G. A. Fredricks and R. B. Nelsen, "On the relationship between Spearman's rho and Kendall's tau for pairs of continuous random variables," Journal of Statistical Planning and Inference, vol. 137, no. 7, pp. 2143-2150, 2007.

[73] V. Schmitz, "Revealing the dependence structure between $x_{(1)}$ and $x_{(n)}$," Journal of Statistical Planning and Inference, vol. 123, no. 1, pp. 41-47, 2004.

[74] X. Li and Z. Li, "Proof of a conjecture on Spearman's $\rho$ and Kendall's $\tau$ for sample minimum and maximum," Journal of Statistical Planning and Inference, vol. 137, no. 1, pp. 359-361, 2007.

[75] Y. P. Chen, "A note on the relationship between Spearman's $\rho$ and Kendall's $\tau$ for extreme order statistics," Journal of Statistical Planning and Inference, vol. 137, no. 7, pp. 2165-2171, 2007. 
[76] J. Navarro and N. Balakrishnan, "Study of some measures of dependence between order statistics and systems," Journal of Multivariate Analysis, vol. 101, no. 1, pp. 52-67, 2010.

[77] T. Hu and C. Xie, "Negative dependence in the balls and bins experiment with applications to order statistics," Journal of Multivariate Analysis, vol. 97, no. 6, pp. 1342-1354, 2006.

[78] D. Dubhashi and O. Häggström, "A note on conditioning and stochastic domination for order statistics," Journal of Applied Probability, vol. 45, no. 2, pp. 575-579, 2008.

[79] T. Hu and H. Chen, "Dependence properties of order statistics," Journal of Statistical Planning and Inference, vol. 138, no. 7, pp. 2214-2222, 2008.

[80] W. Zhuang, J. Yao, and T. Hu, "Conditional ordering of order statistics," Journal of Multivariate Analysis, vol. 101, no. 3, pp. 640-644, 2010.

[81] J. Lynch, G. Mimmack, and F. Proschan, "Uniform stochastic orderings and total positivity," The Canadian Journal of Statistics, vol. 15, no. 1, pp. 63-69, 1987.

[82] W. Chan, F. Proschan, and J. Sethuraman, "Convex-ordering among functions, with applications to reliability and mathematical statistics," in Topics in Statistical Dependence, vol. 16 of IMS Lecture Notes Monograph Series, pp. 121-134, Institute of Mathematical Statistics, Hayward, Calif, USA, 1990.

[83] R. E. Lillo, A. K. Nanda, and M. Shaked, "Preservation of some likelihood ratio stochastic orders by order statistics," Statistics and Probability Letters, vol. 51, no. 2, pp. 111-119, 2001.

[84] N. Torrado and J. J. P. Veerman, "Asymptotic reliability theory of $k$-out-of- $n$ systems," Journal of Statistical Planning and Inference, vol. 142, no. 9, pp. 2646-2665, 2012.

[85] S. C. Kochar and M. Xu, "Stochastic comparisons of parallel systems when components have proportional hazard rates," Probability in the Engineering and Informational Sciences, vol. 21, no. 4, pp. 597-609, 2007.

[86] J. L. Bon and E. Păltănea, "Comparison of order statistics in a random sequence to the same statistics with I.I.D. variables," ESAIM-Probability and Statistics, vol. 10, pp. 1-10, 2006.

[87] S. C. Kochar and M. Xu, "Comparisons of parallel systems according to the convex transform order," Journal of Applied Probability, vol. 46, no. 2, pp. 342-352, 2009.

[88] S. C. Kochar and M. Xu, "On the skewness of order statistics with applications," Annals of Operations Research. In press.

[89] E. Păltănea, "On the comparison in hazard rate ordering of fail-safe systems," Journal of Statistical Planning and Inference, vol. 138, no. 7, pp. 1993-1997, 2008.

[90] P. Zhao and N. Balakrishnan, "Characterization of MRL order of fail-safe systems with heterogeneous exponential components," Journal of Statistical Planning and Inference, vol. 139, no. 9, pp. 3027$3037,2009$.

[91] P. Zhao, X. Li, and N. Balakrishnan, "Likelihood ratio order of the second order statistic from independent heterogeneous exponential random variables," Journal of Multivariate Analysis, vol. 100, no. 5, pp. 952-962, 2009.

[92] P. Zhao and N. Balakrishnan, "Dispersive ordering of fail-safe systems with heterogeneous exponential components," Metrika, vol. 74, no. 2, pp. 203-210, 2011.

[93] T. Hu, "Monotone coupling and stochastic ordering of order statistics," Journal of Systems Science and Complexity, vol. 8, no. 3, pp. 209-214, 1995.

[94] L. Sun and X. Zhang, "Stochastic comparisons of order statistics from gamma distributions," Journal of Multivariate Analysis, vol. 93, no. 1, pp. 112-121, 2005.

[95] B.-E. Khaledi and S. C. Kochar, "Weibull distribution: some stochastic comparisons results," Journal of Statistical Planning and Inference, vol. 136, no. 9, pp. 3121-3129, 2006.

[96] E. W. Stacy, "A generalization of the gamma distribution," Annals of Mathematical Statistics, vol. 33, no. 3, pp. 1187-1192, 1962.

[97] B.-E. Khaledi, S. Farsinezhad, and S. C Kochar, "Stochastic comparisons of order statistics in the scale model," Journal of Statistical Planning and Inference, vol. 141, no. 1, pp. 276-286, 2011.

[98] V. Bagdonavicius and M. Nikulin, Accelerated Life Models, Chapman \& Hall/CRC, Boca Raton, Fla, USA, 2002.

[99] N. Balakrishnan and P. Zhao, "Hazard rate comparison of parallel systems with heterogeneous gamma components," Journal of Multivariate Analysis, vol. 113, pp. 153-160.

[100] Y. S. Sathe, "On the correlation coefficient between the first and the $r$-th smallest order statistics based on $n$ independent exponential random variables," Communications in Statistics, vol. 17, pp. 3295-3299, 1988.

[101] S. R. Jammalamadaka and E. Taufer, "Testing exponentiality by comparing the empirical distribution function of the normalized spacings with that of the original data," Journal of Nonparametric Statistics, vol. 15, no. 6, pp. 719-729, 2003. 
[102] S. R. Jammalamadaka and M. N. Goria, "A test of goodness-of-fit based on Gini's index of spacings," Statistics and Probability Letters, vol. 68, no. 2, pp. 177-187, 2004.

[103] M. Xu and X. Li, "Some further results on the winner's rent in the secondprice business auction," Sankhya, vol. 70, no. 1, pp. 124-133, 2008.

[104] R. E. Barlow and F. Proschan, "Inequalities for linear combinations of order statistics from restricted families," Annals of Mathematical Statistics, vol. 37, pp. 1574-1592, 1966.

[105] N. Misra and E. C. van der Meulen, "On stochastic properties of m-spacings," Journal of Statistical Planning and Inference, vol. 115, no. 2, pp. 683-697, 2003.

[106] N. Torrado, R. E. Lillo, and M. P. Wiper, "On the conjecture of Kochar and Korwar," Journal of Multivariate Analysis, vol. 101, no. 5, pp. 1274-1283, 2010.

[107] S. Wen, Q. Lu, and T. Hu, "Likelihood ratio orderings of spacings of heterogeneous exponential random variables," Journal of Multivariate Analysis, vol. 98, no. 4, pp. 743-756, 2007.

[108] M. Xu, X. Li, P. Zhao, and Z. Li, "Likelihood ratio order of m-spacings in multiple-outlier models," Communications in Statistics, vol. 36, no. 8, pp. 1507-1525, 2007.

[109] H. Chen and T. Hu, "Multivariate likelihood ratio orderings between spacings of heterogeneous exponential random variables," Metrika, vol. 68, no. 1, pp. 17-29, 2008.

[110] S. C. Kochar, X. Li, and M. Xu, "Excess wealth order and sample spacings," Statistical Methodology, vol. 4, no. 4, pp. 385-392, 2007.

[111] F. Belzunce, "On a characterization of right spread order by the increasing convex order," Statistics and Probability Letters, vol. 45, no. 2, pp. 103-110, 1999.

[112] S. C. Kochar and M. Xu, "Stochastic comparisons of spacings from heterogeneous samples," in Advanced in Directional and Linear Statistics: A Festschrift for Sreenivasa Rao Jammalamadaka, M. Wells and A. Sengupta, Eds., pp. 113-1129, Springer, New York, NY, USA, 2011.

[113] N. Balakrishnan and M. Xu, "On the sample ranges from heterogeneous exponential variables," Journal of Multivariate Analysis, vol. 109, pp. 1-9, 2012.

[114] N. Torrado and R. E. Lillo, "Likelihood ratio order of spacings from two heterogeneous samples," Journal of Multivariate Analysis, vol. 114, pp. 338-348, 2013.

[115] W. Ding, G. Da, and P. Zhao, “On sample ranges from two heterogeneous random variables," Journal of Multivariate Analysis.

[116] B.-E. Khaledi and S. C. Kochar, "Dependence among spacings," Probability in the Engineering and Informational Sciences, vol. 14, no. 4, pp. 461-472, 2000.

[117] B. Epstein, "Estimation from life test data," Technometric, vol. 2, no. 4, pp. 447-454, 1960. 


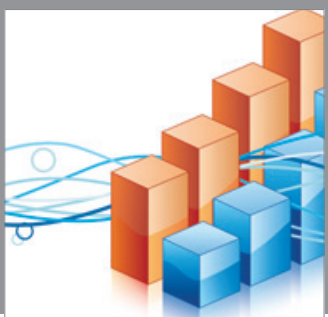

Advances in

Operations Research

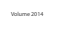

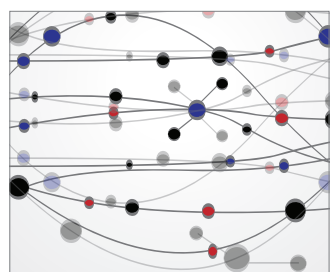

\section{The Scientific} World Journal
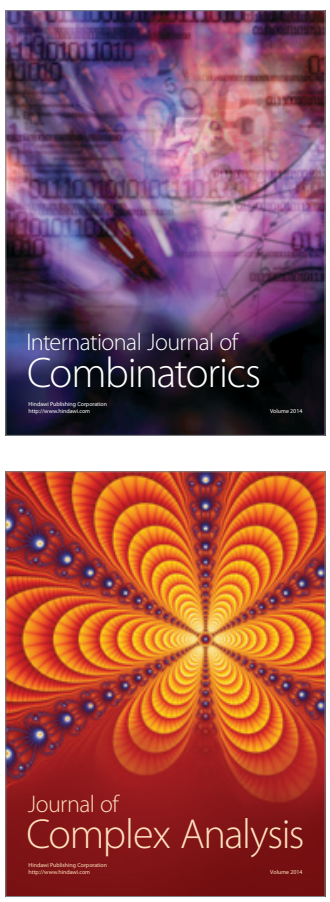

International Journal of

Mathematics and

Mathematical

Sciences
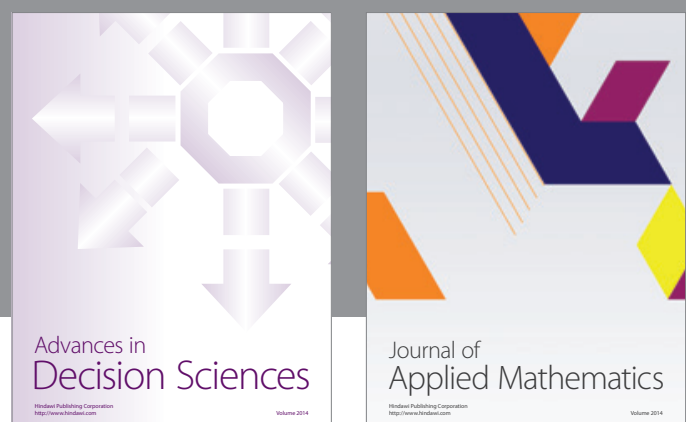

Journal of

Applied Mathematics
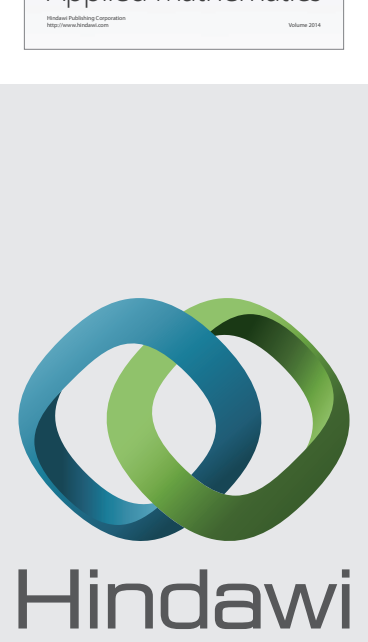

Submit your manuscripts at http://www.hindawi.com
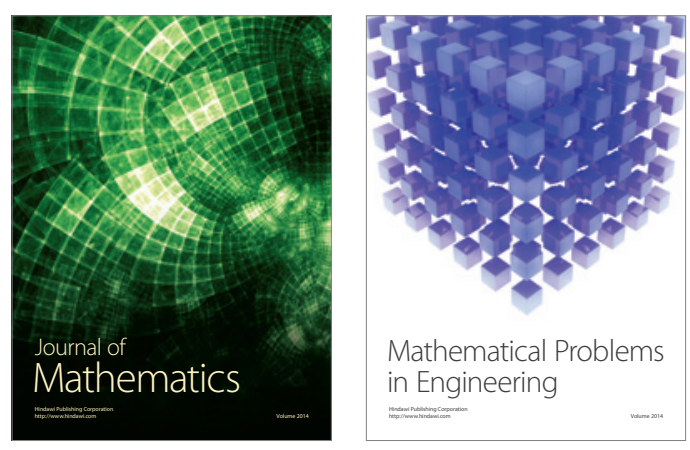

Mathematical Problems in Engineering
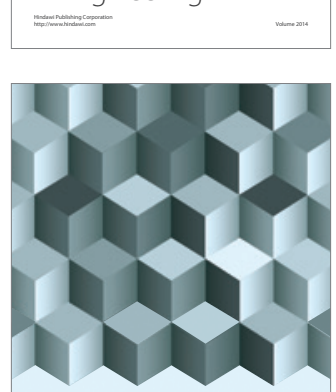

Journal of

Function Spaces
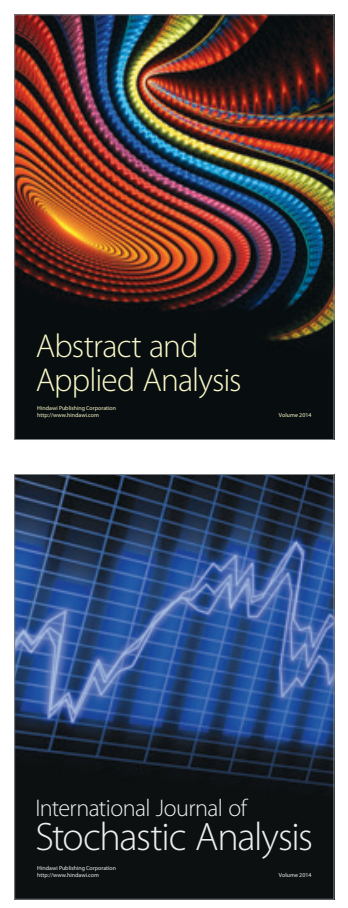

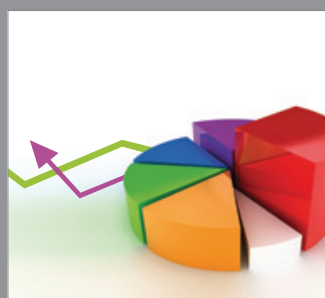

ournal of

Probability and Statistics

Promensencen
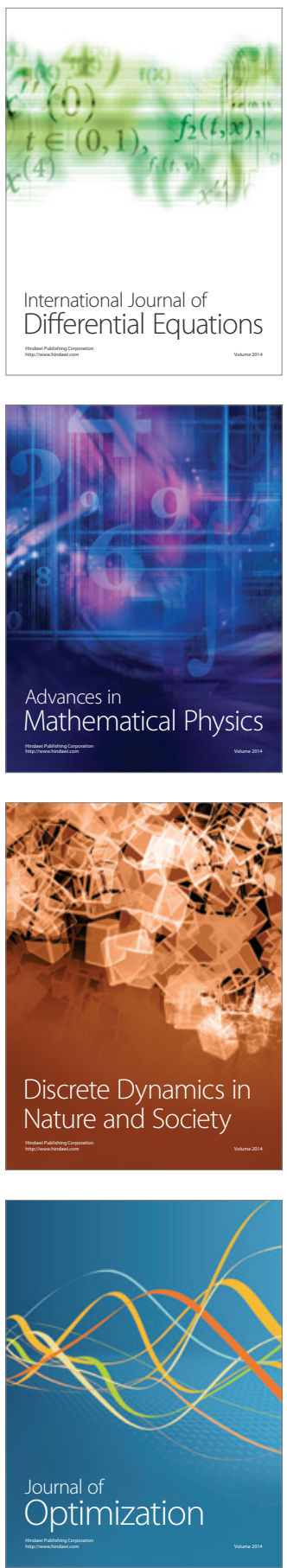